Francielly Antonio Monteiro

\title{
IMPACTO DO NÍVEL DE ATIVIDADE FÍSICA SOBRE OS INDICADORES DE RISCO CARDIOVASCULAR EM PACIENTES COM DOENÇA ARTERIAL OBSTRUTIVA PERIFÉRICA: estudo observacional longitudinal
}

Dissertação apresentada à Faculdade
Israelita de Ciências da Saúde Albert
Einstein para obtenção do Título de Mestre
em Ciências da Saúde.

São Paulo 
Francielly Antonio Monteiro

\title{
IMPACTO DO NÍVEL DE ATIVIDADE FÍSICA SOBRE OS INDICADORES DE RISCO CARDIOVASCULAR EM PACIENTES COM DOENÇA ARTERIAL OBSTRUTIVA PERIFÉRICA: estudo observacional longitudinal
}

\author{
Dissertação apresentada à Faculdade \\ Israelita de Ciências da Saúde Albert \\ Einstein para obtenção do Título de Mestre \\ em Ciências da Saúde.
}

Orientador: Prof. Dr. Gabriel Grizzo Cucato

São Paulo 
M775i

Monteiro, Francielly Antonio

Impacto do nível de atividade física sobre os indicadores de risco cardiovascular em pacientes com doença arterial obstrutiva periférica : estudo observacional longitudinal / Francielly Antonio Monteiro. -- São Paulo, 2019.

xii, $37 \mathrm{f}$.

Dissertação (Mestrado) - Faculdade Israelita de Ciências da Saúde Albert Einstein. Instituto Israelita de Ensino e Pesquisa Albert Einstein. Programa de Pós-Graduação em Ciências da Saúde.

Título em inglês: Impact of physical activity level on cardiovascular risk indicators in patients with peripheral obstructive arterial disease: Iongitudinal observational study.

1. Doença arterial periférica. 2. Sistema cardiovascular. 3. Pressão arterial. 4. Atividade motora.

NLM - WG 550

Elaborada pelo Sistema Einstein Integrado de Bibliotecas 
FACULDADE ISRAELITA DE CIÊNCIAS DA SAÚDE ALBERT EINSTEIN

Coordenador do Curso de Pós-Graduação: Prof. Dr. Luiz Vicente Rizzo 
Francielly Antonio Monteiro

\section{IMPACTO DO NÍVEL DE ATIVIDADE FÍSICA SOBRE OS INDICADORES DE RISCO CARDIOVASCULAR EM PACIENTES COM DOENÇA ARTERIAL OBSTRUTIVA PERIFÉRICA: estudo observacional longitudinal}

Presidente da banca: Prof. Dr. Gabriel Grizzo Cucato

\section{BANCA EXAMINADORA}

Membros titulares:

Prof. Dr. Nelson Wolosker

Prof. Dr. Hélcio kanegusukuProf.

Dr. Marcelo Passos Teivelis

Membros suplentes:

Profa. Dra. Luciana Diniz Nagem Janot de Matos

Prof. Dr. Danilo Sales Bocalini

Aprovada em 16/12/2019. 


\section{Agradecimentos}

Primeiramente eu gostaria de agradecer a Deus por todas as oportunidades concedidas e por ter colocado pessoas maravilhosas em toda a jornada da minha vida.

Aos meus pais, Oseas e Ester, por sempre me apoiarem e me mostrarem que o estudo é o melhor caminho a ser seguido.

A minha tia, Lúcia, que não está mais presente neste mundo, mas sempre acreditou no meu potencial.

A minha grande amiga, Larissa, que nos momentos mais difíceis dessa jornada esteve ao meu lado, me incentivando a continuar com a cabeça erguida.

Aos meus professores da graduação, Prof. Dr. Danilo Bocalini, Prof. Dr. Alexandre Evangelista, Prof. Dr. Andrey Jorge Serra, Profa. Dra. Aletha Caetano, Profa. Adriana Sbampato, Profa. Dra. Tatiana Freitas e Profa. Dra. Érika Joaquim por todo ensinamento e incentivo recebido ao longo dos anos.

Aos meus amigos, Cícera, Rafael, Mauro e Molina por terem sido compreensíveis quando eu me abdicava de sair com eles para me dedicar ao mestrado e, claro, pelo apoio recebido de cada um deles.

Aos meus queridos alunos e ex-alunos, que sempre depositaram confiança em meu trabalho.

A Profa. Dra. Carla de Sá Pinto, que sempre esteve disposta a ajudar os seus alunos durante a jornada de formação.

A todos os integrantes do grupo GEPICARDIO, que foram essenciais para que este trabalho pudesse acontecer.

Ao meu querido amigo, Samuel Amorin, na qual tive o prazer de dividir os momentos bons e ruins nesses dois anos de mestrado.

A equipe do Ambulatório de Cirurgia Vascular do Hospital das Clínicas da Faculdade de Medicina da Universidade de São Paulo por cederem o espaço para que este trabalho pudesse ser concluído.

Aos meus pacientes por se disponibilizarem a participar deste estudo e contribuir com a ciência e a sociedade. 
Por fim, ao meu orientador Dr. Gabriel, que apesar da pouca diferença de idade, eu o chamo de meu pai científico, por ter me ensinado muita coisa nesses três anos de orientação, pelos conselhos, paciência e por sua amizade. 
"Uma vida sem desafios não vale a pena ser vivida."

Sócrates

vii 


\section{Sumário}

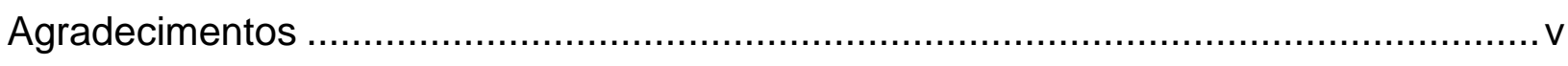

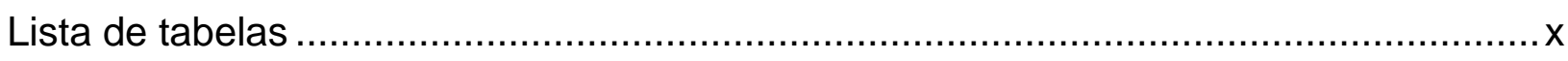

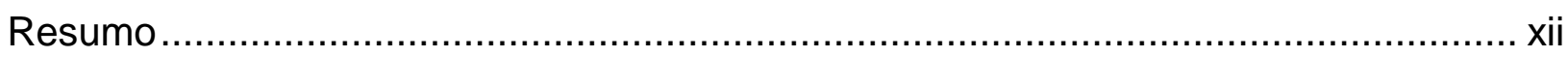

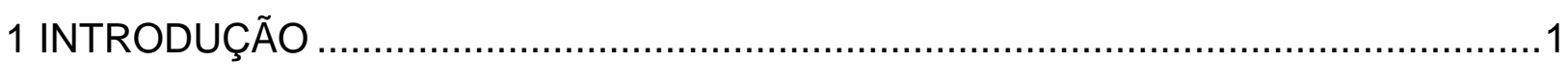

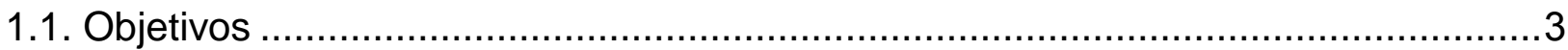

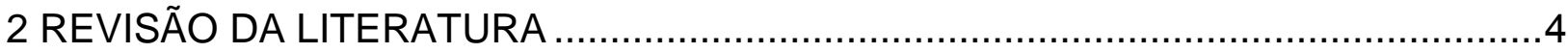

2.1 Doença arterial obstrutiva periférica .....................................................

2.1.1 Prevalência..................................................................................... 4

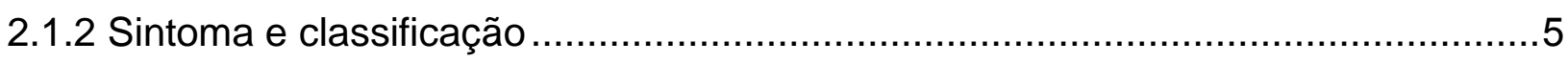

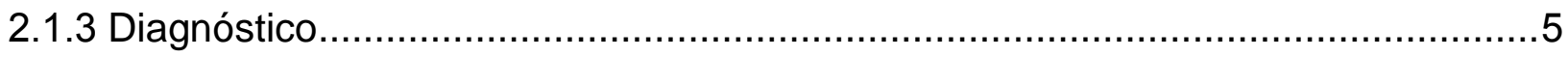

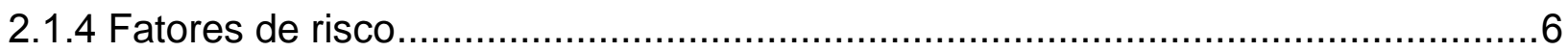

2.1.5 Consequências da doença arterial obstrutiva periférica ................................ 7

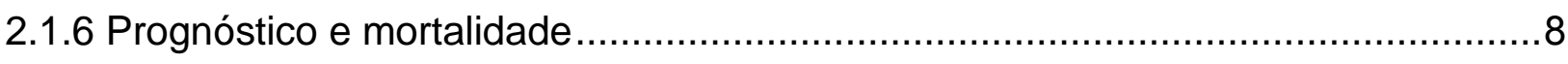

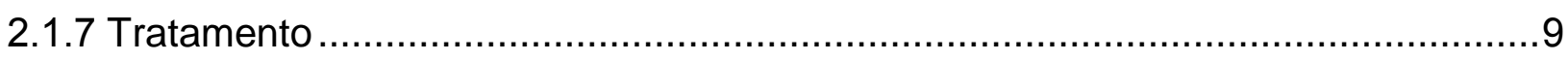

2.2 Exercício como tratamento da doença arterial obstrutiva periférica ......................10

2.2.1 Exercício como tratamento inicial ........................................................ 10

2.2.2 Efeitos do exercício físico na função cardiovascular em pacientes com doença

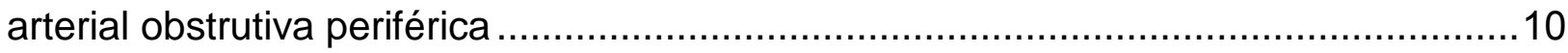

2.2.2.1 Efeitos do exercício físico na pressão arterial .......................................11

2.2.2.2 Efeitos do exercício físico na rigidez arterial ........................................ 11

2.2.2.3 Efeitos do exercício físico na modulação autonômica cardíaca ........................12

2.2.2.4 Efeitos do exercício físico no fluxo sanguíneo e na capacidade vasodilatadora12

2.3 Nível de atividade física de pacientes com doença arterial obstrutiva periférica.....12

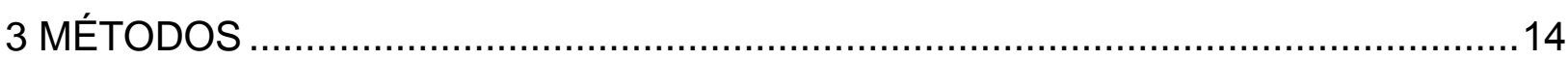

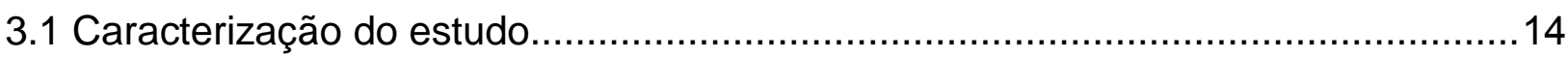

3.2 Recrutamento, triagem e dimensionamento da amostra ................................ 14

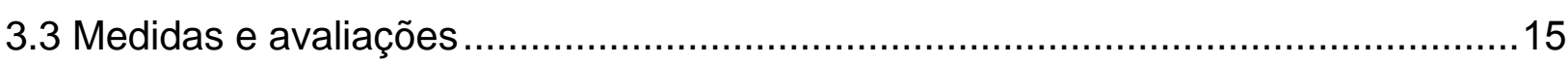

3.4 Variável independente - nível de atividade física........................................ 15

3.5 Desfechos primários - indicadores de risco cardiovascular................................ 16

3.5.1 Pressão arterial clínica ................................................................... 16

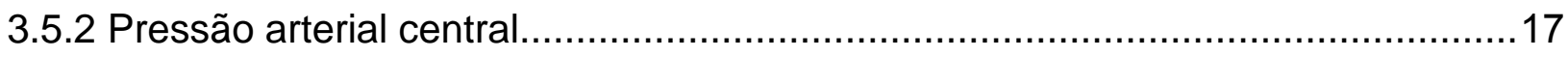


3.5.3 Modulação autonômica do sistema cardiovascular 17

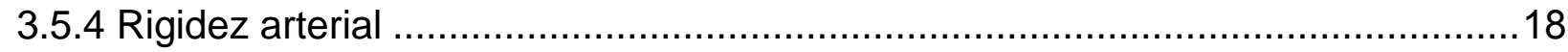

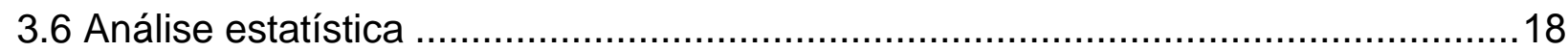

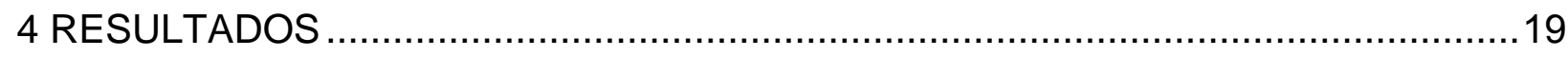

5 DISCUSSÃO

5.1 Nível de atividade física no baseline e follow-up de 2 anos .................................25

5.2 Modificações nas características clínicas no baseline e follow-up de 2 anos ..........25 5.3 Modificações nos indicadores de risco cardiovascular no baseline e follow-up de 2 anos 26

5.4 Influência do nível de atividade física sobre os indicadores de risco cardiovascular

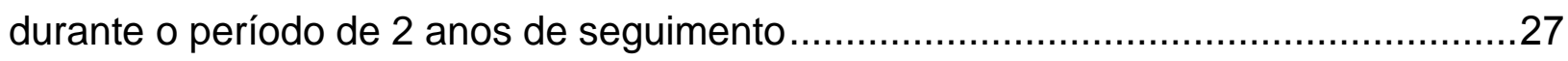

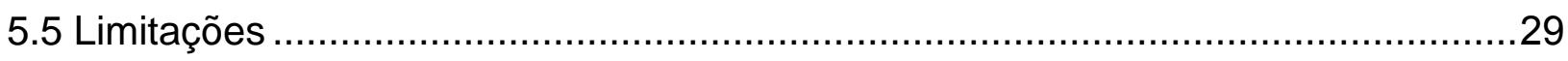

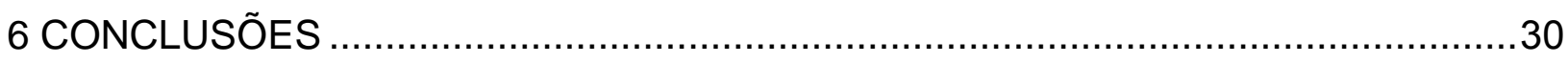

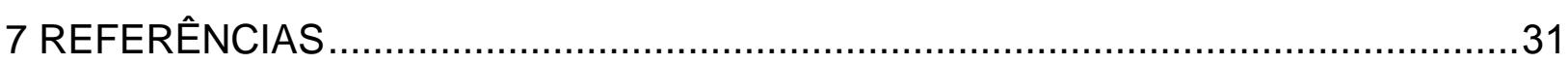

Abstract 


\section{Lista de figuras}

Figura 1. Prognóstico dos pacientes com doença arterial obstrutiva periférica ..............2 Figura 2. Razão de chance para doença arterial obstrutiva periférica em países de renda alta e países de renda média e baixa ......................................................... 7

Figura 3. Desenho experimental do estudo ................................................... 14

Figura 4. Tercis de acordo com a atividade física total .....................................16

Figura 5. Fluxograma dos pacientes envolvidos no estudo ................................. 19 


\section{Lista de tabelas}

Tabela 1. Nível de atividade física de pacientes no baseline e follow-up. 19

Tabela 2. Características clínicas e fatores de risco de pacientes com claudicação intermitente no baseline e follow-up

Tabela 3. Pressão arterial e frequência cardíaca dos pacientes no baseline e follow-up .21

Tabela 4. Indicadores de rigidez arterial e onda refletida de pacientes no baseline e follow-up. .21

Tabela 5. Variáveis de análise da modulação autonômica cardíaca no baseline e follow-up. .22

Tabela 6. Características clínicas e fatores de risco de acordo com os deltas dos tercis do tempo despendido em atividade física total. .22

Tabela 7. Alterações $(\Delta)$ nas variáveis cardiovasculares de acordo com as alterações na atividade física total .23 


\section{Resumo}

Introdução: Pacientes com doença arterial obstrutiva periférica têm redução da capacidade funcional e alto risco de eventos cardiovasculares fatais e não fatais. Apesar de atividade física ser uma abordagem terapêutica para melhorar a capacidade funcional, pouco se sabe sobre os seus possíveis efeitos positivos em indicadores de risco cardiovascular de forma longitudinal. Objetivos: Analisar longitudinalmente a influência do nível de atividade física sobre os indicadores de risco cardiovascular em pacientes com doença arterial obstrutiva periférica. Métodos: Esse estudo teve início no ano de 2015. Na primeira fase, 226 pacientes foram recrutados. Na segunda fase, após 2 anos, 77 pacientes aceitaram o convite para retornarem e foram submetidos a todas as avaliações realizadas na primeira fase. Foram avaliados os níveis de atividade física (acelerometria) e indicadores de risco cardiovascular (pressão arterial, modulação autonômica cardíaca e rigidez arterial). As estatísticas descritivas para variáveis contínuas foram expressas como média, desvio-padrão e $95 \%$ de intervalo de confiança (IC 95\%). As variáveis categóricas foram apresentadas como percentual e seu intervalo de confiança de $95 \%$. As comparações de médias ao longo do tempo foram analisadas por meio de análise de variância para medidas repetidas com o teste post-hoc de Bonferroni. O nível de significância adotado foi $5 \%$. Resultados: Pacientes com doença arterial obstrutiva periférica apresentaram piora da rigidez arterial central, índice tornozelo-braquial e o desvio padrão de todos os intervalos $R R(p<0,05)$. Além disso, os pacientes reduziram seus níveis de atividade física total $(p<0,05)$ no seguimento de 2 anos. Por outro lado, não houve alterações nos indicadores de risco cardiovasculares no tempo despendido em atividade física total $(p>0,05)$. Conclusões: Em pacientes com doença arterial obstrutiva periférica, foram encontradas reduções dos níveis de atividade física total. Entretanto, não houve influência do nível de atividade física total nas alterações dos indicadores de risco cardiovascular em pacientes com doença arterial obstrutiva periférica após o seguimento de 2 anos.

Descritores: Doença arterial obstrutiva periférica; Sistema cardiovascular; Pressão arterial; Atividade motora 


\section{INTRODUÇÃO}

A doença arterial obstrutiva periférica (DAOP) é resultado de um processo aterosclerótico crônico, que de forma progressiva, leva a obstrução parcial ou total das artérias que irrigam as regiões dos membros inferiores ou superiores do corpo humano. ${ }^{(1)}$ No Brasil a DAOP afeta $10,5 \%$ dos indivíduos com idade acima de 18 anos aumentando sua prevalência nas faixas etárias acima dos 50 anos. ${ }^{(2)} \mathrm{A}$ claudicação intermitente $(\mathrm{Cl})$, o principal sintoma da DAOP, é caracterizada por dor, câimbra, ardência ou formigamento que acomete o(s) membro(s) afetado, geralmente o(s) inferior(es) durante a prática de atividade física, principalmente na forma de caminhada. ${ }^{(3)}$ Pacientes com DAOP e sintomas de $\mathrm{Cl}$ apresentam limitação de locomoção, piora nos indicadores de saúde ${ }^{(4)}$ e comprometimento da qualidade de vida. ${ }^{(5)}$

Uma das possíveis causas dos prejuízos funcionais e dos indicadores de saúde dos pacientes parece envolver a redução dos seus níveis de atividade física diários. Essa suposição se deve ao fato que pacientes com DAOP realizam $36 \%$ menos atividade física em comparação com indivíduos sem a doença. ${ }^{(6)}$ Além disso, pacientes com DAOP insuficientemente ativos apresentam menor capacidade de caminhada do que pacientes com maiores níveis de atividade física. De fato, Gardner et al., em estudo com 133 pacientes com DAOP, observaram que o desempenho de diversas atividades físicas em diferentes cadências esteve correlacionado com a capacidade de caminhada, indicando as relações entre os níveis de atividade física e capacidade funcional. ${ }^{(7)}$

Apesar de a literatura atual apresentar diversos estudos que analisaram a associação entre o nível de atividade física e os indicadores de capacidade funcional de pacientes com DAOP, é interessante notar que, poucos analisaram a associação entre a atividade física e os indicadores de risco cardiovascular. Isso chama a atenção quando levamos em consideração o elevado risco de mortalidade cardiovascular entre pacientes com DAOP quando comparado a seus pares sem a doença (seis a dez vezes maior). ${ }^{\left({ }^{(8)}\right.}$ Ademais, o riscos cardiovasculares dos pacientes com DAOP são maiores do que pacientes com outras doenças ateroscleróticas, tais como a doença arterial coronariana e a doença cerebrovascular. ${ }^{(9)}$ 
Esse dado chama ainda mais a atenção quando se analisa a figura 1. Conforme pode ser observado, o prognóstico dos pacientes com a doença em relação ao membro afetado pela obstrução (lado esquerdo da figura) é menos nocivo do que a evolução do risco cardiovascular desses pacientes (lado direito da figura). De fato, no período de cinco anos, a amputação do membro pode ocorrer em apenas 1-2\% dos casos, ao passo que $50 \%$ dos pacientes apresentam algum evento cardiovascular, sendo $15-30 \%$ desses eventos fatais. ${ }^{(10)}$

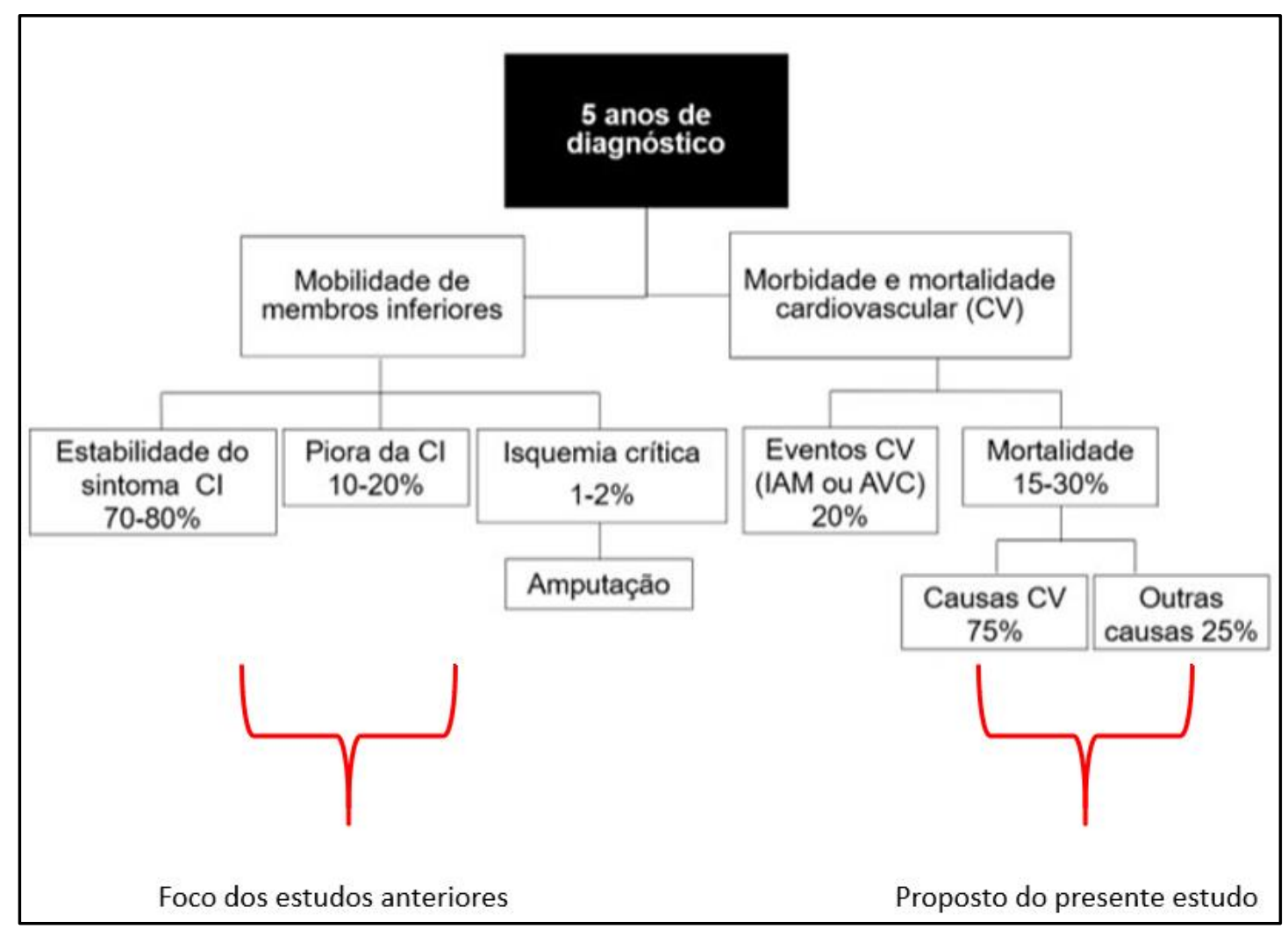

Cl: claudicação intermitente; CV: cardiovascular; IAM: infarto agudo do miocárdio; AVC: acidente vascular cerebral.

Fonte: Adaptado de Weitz JI, Byrne J, Clagett GP, Farkouh ME, Porter JM, Sackett DL, et al. Diagnosis and treatment of chronic arterial insufficiency of the lower extremities: a critical review. Circulation. 1996;94(11):3026-49. ${ }^{(10)}$

Figura 1. Prognóstico dos pacientes com doença arterial obstrutiva periférica

Nesse sentido, alguns estudos em pacientes com DAOP tem demostrado que o exercício físico de forma regular pode ser utilizado como estratégia terapêutica para redução do risco cardiovascular como a diminuição da pressão arterial, aumento da variabilidade da frequência cardíaca e aumento em alguns indicadores de rigidez arterial. ${ }^{(11,12)}$ Todavia, embora programas de intervenção supervisionada venham sendo recomendados, sabe-se que a proporção de pacientes 
engajados nesse tipo de programa ainda é pequena. Dessa forma, alterações no estilo de vida de forma a aumentar os níveis de atividade física habitual têm sido recomendadas. De fato, pacientes com DAOP com maiores níveis de atividade física apresentam maior capacidade funcional e menor mortalidade cardiovascular em comparação a pacientes sedentários. ${ }^{(11,12)}$ Contudo, devido ao delineamento transversal destes estudos, não é possível o estabelecimento de causalidade, sendo que estudos com desenho longitudinais são necessários.

Portanto, o objetivo do estudo foi analisar de forma longitudinal a influência do nível de atividade física sobre indicadores de risco cardiovascular em pacientes com DAOP.

\subsection{Objetivos}

1. Analisar longitudinalmente a influência do nível de atividade física sobre os indicadores de risco cardiovascular em pacientes com doença arterial obstrutiva periférica;

2. Analisar longitudinalmente a influência do nível de atividade física sobre: pressão arterial braquial, pressão arterial central, indicadores de rigidez arterial e modulação autonômica cardíaca. 


\section{REVISÃO DA LITERATURA}

\subsection{Doença arterial obstrutiva periférica}

A DAOP é o resultado de um processo aterosclerótico crônico, que de forma progressiva, causa modificações nas estruturas das artérias, formando placas de ateroma que leva a obstrução parcial ou total das artérias, limitando o fluxo sanguíneo que irriga os membros superiores e/ou inferiores do corpo humano. ${ }^{(13)}$ Com a diminuição do fluxo sanguíneo para as extremidades superiores e inferiores, surge o principal sintoma que pode ser dor, câimbra, formigamento, ardência ou fadiga muscular durante a prática de atividade física. ${ }^{(1)}$ Esse sintoma aparece quando a oferta e demanda de oxigênio é reduzida na musculatura exercitada, ocasionando a isquemia muscular. ${ }^{(4)}$

\subsubsection{Prevalência}

Segundo a American Heart Association, estima-se que 8 a 10 milhões de indivíduos nos Estados Unidos tem a DAOP e esse número pode chegar a afetar 19 milhões de americanos em 2050.(14,15) No Brasil, estima-se que 6 milhões de indivíduos com idades acima de 18 anos apresentam DAOP. ${ }^{(2)}$ Porém, a DAOP é difícil de ser detectada em seu estágio inicial por não apresentar sintomas, dificultando a avaliação da sua real prevalência.

O aumento da prevalência da DAOP está relacionado com o avanço da idade. Um estudo realizado por Norgren et al. ${ }^{(4)}$ demonstrou que a prevalência da DAP na população mundial com idades entre 35-39 anos é de 1\%; 3,1\% com idades entre 60-64 anos e de 7,2\% em idades entre 70-74 anos. ${ }^{(4)}$

Em outro estudo sobre prevalência e idade demonstrou que a prevalência da DAOP entre os sexos em países de alta renda foram semelhantes e aumentam com o avançar da idade, indivíduos entre 45-49 anos tem um aumento de 5\% de chance de desenvolver a DAOP e indivíduos com idades entre 85-89 anos tem um aumento de $18 \%{ }^{(16)}$ As taxas de prevalência nos países de baixa e média renda também aumentam com o avanço da idade, porém, quando as taxas de idade foram comparadas entre os países de alta renda, a prevalência da DAOP foi maior em 
mulheres do que nos homens $(6,3 \%$ e $2,9 \%$ aos $45-49$ anos em comparação com $12,3 \%$ e $10,1 \%$ aos $75-79$ anos)..$^{(17)}$

\subsubsection{Sintoma e classificação}

A DAOP pode ser classificada em 4 estágios de evolução. Essas classificações permitem identificar os seus sinais e sintomas que se tornam mais graves com o avanço da doença. De acordo com os critérios de Fontaine, os estágios da doença são: ${ }^{(18)}$

- Estágio 1: não apresentam sintomas;

- Estágio 2: surgimento de dores nos membros inferiores durante a prática de atividade física (claudicação intermitente);

- Estágio 3: dores no membro afetado surgem em repouso;

- Estágio 4: isquemia crítica, aumentando as chances de amputação do membro afetado.

Pacientes que se encontram no estágio 2 da doença apresentam o principal sintoma, a Cl. ${ }^{(1)}$ Como citado anteriormente, a $\mathrm{Cl}$ é um dos sintomas da DAOP, que se manifesta comumente como dor, ardência, câimbra e formigamentos devido ao fluxo sanguíneo inadequado aos tecidos periféricos que estão sendo executados durante a prática de atividade física, podendo ser cessada durante 0 repouso. ${ }^{(1)} \mathrm{A} \mathrm{Cl}$ é encontrada em um a cada três ou quatro pacientes diagnosticados com DAOP. ${ }^{(4,19)}$

\subsubsection{Diagnóstico}

Para o diagnóstico da DAOP, podem ser realizados exames como a ultrassonografia duplex, arteriografia, angioressonância nuclear magnética e a angiotomográfia computadorizada, que são considerados exames mais sofisticados, possibilitando revelar o tamanho da lesão oclusiva nas artérias acometidas pela doença.

Contudo, um dos exames mais utilizados é o índice tornozelo braço (ITB) que identifica a pressão das artérias pediosa e tibiais dos membros inferiores. ${ }^{(20)}$ Para mensurar o valor do ITB, é utilizado a aferição da pressão arterial sistólica dos 
membros inferiores (ambos tornozelos) e dividida pelo maior valor da pressão arterial sistólica do braço (ambos os braços). ${ }^{(21)}$ São considerados como presença de DAOP, indivíduos que apresentem valores de ITB $<0,90 .{ }^{(22)} O$ valor do ITB também determina a gravidade da obstrução, podendo ser classificadas como:(1,23)

- 1,00 a 1,29: normal;

- 0,91 a 0,99: limítrofe;

- 0,41 a 0,90: DAOP leve a moderada;

- 0,00 a 0,40: DAOP grave.

\subsubsection{Fatores de risco}

Estudos epidemiológicos apontam que a alta prevalência de hipertensão arterial, dislipidemia, diabetes mellitus, faixa etária, tabagismo, histórico de doenças cardiovasculares estão relacionados com o surgimento e agravamento da DAOP. ${ }^{(24)}$

O tabagismo é o principal fator de risco para o surgimento da DAOP, dobrando o risco de ter a doença em comparação com indivíduos que não fumam. ${ }^{(24)}$ Quanto maior é a quantidade e o tempo de tabagismo, maiores serão os riscos do surgimento da DAOP e ter piora do diagnóstico. ${ }^{(25)}$

Alguns estudos epidemiológicos demonstraram que indivíduos com diabetes mellitus tem maiores chances de apresentar DAOP (assintomática e sintomática), podendo ser aumentada em $26 \%$ para cada aumento do nível de hemoglobina A1c. ${ }^{(24,25)}$ Para aqueles indivíduos com diagnóstico de DAOP e diabetes mellitus, estes apresentam duas vezes mais chances em apresentar $\mathrm{Cl}$ em comparação com indivíduos sem diabetes mellitus. ${ }^{(25)}$

A hipertensão arterial também tem sido relatada em alguns estudos como um fator de risco para o desenvolvimento da DAOP, porém, quando comparada com o tabagismo e o diabetes mellitus, sua relação é modesta. ${ }^{(25)}$ Além disso, alguns estudos têm mostrado que os fatores de riscos acumulativos aumentam as chances de DAOP, ou seja, quanto maior for o número de doenças que um indivíduo tenha, maiores as chances de desenvolver a DAOP. ${ }^{(26,27)}$

Níveis altos de colesterol total, lipoproteínas de baixa densidade (LDL), triglicerídeos e lipoproteínas também estão associados ao fator de risco para o 
surgimento da DAOP. Por outro lado, níveis elevados de lipoproteínas de alta densidade (HDL) estão relacionados com o efeito protetor para o desenvolvimento da DAOP. ${ }^{(28)}$

Apesar de diversos fatores estarem relacionados a DAOP, como citado anteriormente, o tabagismo e o diabetes mellitus são os mais fortes indicadores para a doença, aumentando em 2,5 vezes de chance de mortalidade e morbidade por DAOP. ${ }^{(29)} \mathrm{Na}$ figura 2 se encontram os principais fatores de risco para a DAOP. ${ }^{(24)}$

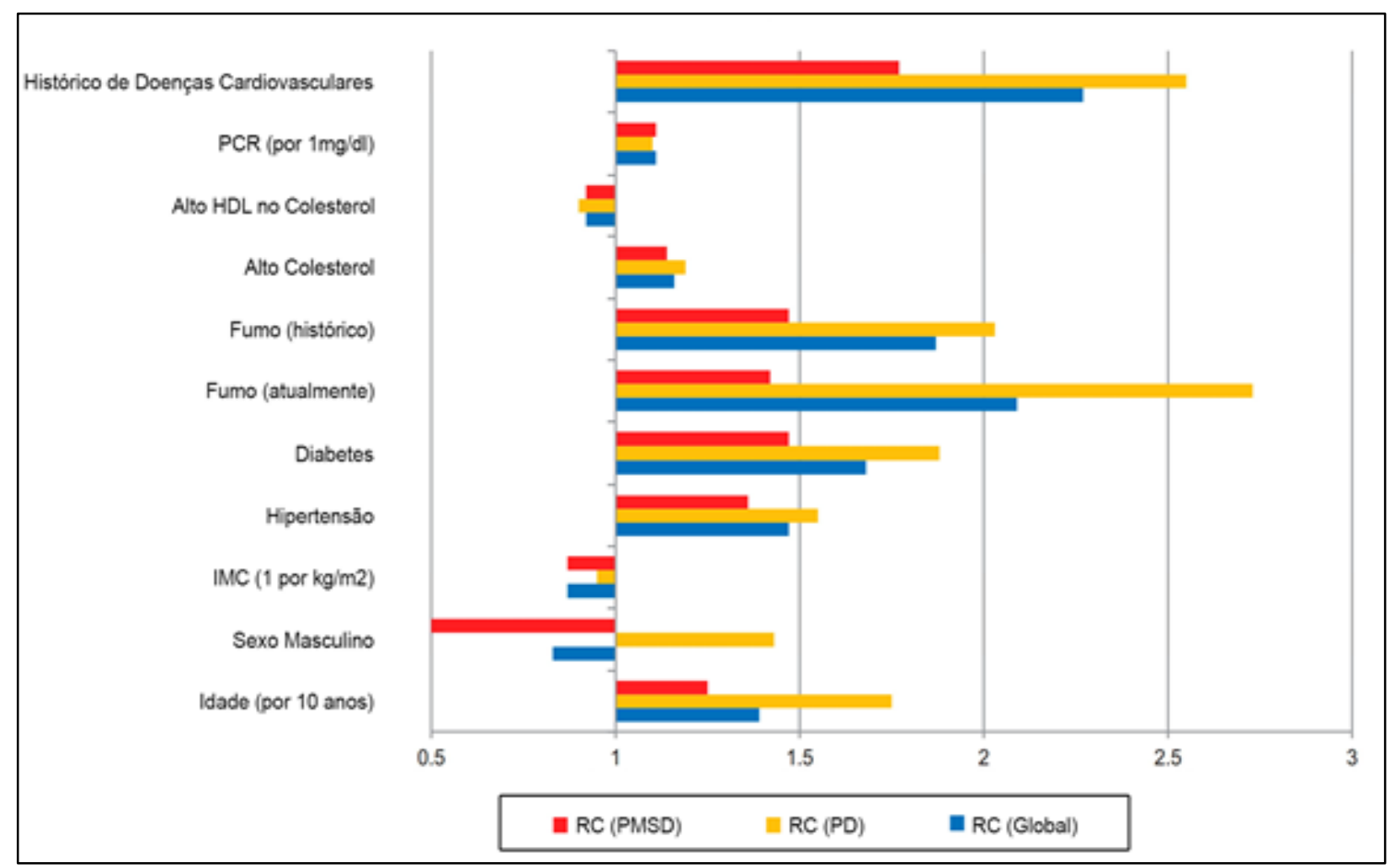

RC: razão de chance; PD: países desenvolvidos; PMSD: países subdesenvolvidos; IMC: índice de massa corporal; DC: doenças cardiovasculares; PCR: proteína c reativa e HDL.

Fonte: Traduzido de Criqui $\mathrm{MH}$, Aboyans V. Epidemiology of peripheral artery disease. Circ Res. 2015;116(9):1509-26. Figure 3, Odds ratios for peripheral artery disease in high-income countries (HIC) and low- to middle income countries (LMIC); p. 1518. ${ }^{(24)}$

Figura 2. Razão de chance para doença arterial obstrutiva periférica em países de renda alta e países de renda média e baixa

\subsubsection{Consequências da doença arterial obstrutiva periférica}

Pelo fato de apresentarem sintoma de dor durante a prática de atividade física, pacientes com DAOP tem redução em seus níveis de atividade física, comprometendo diversos atributos da aptidão física e a qualidade de vida. ${ }^{(3-5)}$ Estudo recente do nosso grupo demonstrou que pacientes com DAOP despendem em média 
$640 \pm 121 \mathrm{~min} / \mathrm{dia}, 269 \pm 94 \mathrm{~min} / \mathrm{dia}, 36 \pm 27 \mathrm{~min} / \mathrm{dia}$ e $15 \pm 16 \mathrm{~min} / \mathrm{dia}$ em atividades sedentárias, muito baixa, baixa e moderada/vigora, respectivamente. ${ }^{(30)}$ Além disso, apenas $3,4 \%$ dos pacientes incluídos no estudo atingiram as recomendações mínimas para a prática de atividade física de acordo com a recomendação da organização mundial da saúde, demostrando a baixa realização de atividade física nessa população. ${ }^{(30)}$

Adicionalmente, um estudo realizado por McDermott et al. ${ }^{(31)}$ demonstrou que indivíduos com DAOP que gastam mais tempo (horas) em comportamento sedentário (sentado e deitado) apresentaram maiores declínios no desempenho da caminhada nos testes de 6 minutos e 4 metros.

De fato, a isquemia crônica leva a reduções nos níveis de atividade física de pacientes com DAOP, contribuindo com o agravamento da doença. Como consequência, pacientes com DAOP tem alterações estruturais no músculo esquelético, incluindo perda de força e massa muscular (perda de miócitos, reduções das fibras tipo II, aumento da fibrose, alterações nos capilares, deposição de gordura) do músculo gastrocnêmio, trazendo mais dificuldades e comprometimento da caminhada. $^{(32-34)}$

Além disso, alterações no número de mitocôndrias têm sido relatadas em pacientes com DAOP e tem sido associada ao aumento da inflamação sistêmica a síntese proteica muscular, ocasionando disfunção mitocondrial e muscular. ${ }^{(34)}$

\subsubsection{Prognóstico e mortalidade}

Pacientes diagnosticados com a DAOP podem ter obstruções em outras regiões do corpo humano, além do membro afetado, artérias que irrigam o cérebro e o coração podem sofrer modificações, aumentando o risco de mortalidade.

Ao longo dos anos, com a evolução da DAOP, pode trazer um risco aumentado de amputação do membro e/ou alto risco de mortalidade por evento cardiovascular. ${ }^{(1,4)}$ Como mostrado anteriormente na figura 1 , no primeiro ano após o diagnóstico da doença, cerca de $25 \%$ dos pacientes com DAOP terão o seu estado clínico piorado. ${ }^{(2)} \mathrm{E}$ os dados chamam mais atenção quando após 5 anos de diagnóstico, a amputação do membro afetado pela doença atinge em torno de 1-2\% 
dos pacientes, ao passo que $50 \%$ deles apresentam algum evento cardiovascular, sendo $15-30 \%$ desses eventos fatais. ${ }^{(10)}$

Somando-se aos dados apresentados anteriormente, um estudo realizado por Criqui et al. ${ }^{(24)}$ observou durante 10 anos que pacientes com DAOP tinham 10 a 15 vezes o risco aumentado de morte por doenças cardiovasculares, independente da gravidade da doença. Em outro estudo em pacientes com DAOP demonstrou que $40 \%-60 \%$ das mortes foram causadas por doenças da artéria do coração, $10-20 \%$ por doenças das artérias cerebrais e 10\% foram ocasionadas por outras doenças cardiovasculares, apenas $30 \%$ das mortes não estavam relacionadas com eventos cardiovasculares. ${ }^{(25)}$

Desta maneira, os dados demonstram que o risco de ter o membro afetado amputado é muito menor em comparação com o alto risco de mortalidade por evento cardiovascular nessa população. ${ }^{(35,36)}$

Além disso, alguns estudos demostram que pacientes com DAOP apresentam piora na função e na regulação cardiovascular quando comparados com indivíduos sem a doença, apresentando:

1. Maior rigidez arterial devido ao processo aterosclerótico que afeta as estruturas arteriais, prejudicando ainda mais a severidade da doença; ${ }^{(17,37-39)}$

2. Maior modulação simpática e menor modulação parassimpática para o coração, e: ${ }^{(40)}$

3. Redução na capacidade vasodilatadora, agravando a função vascular. ${ }^{(40)}$

\subsubsection{Tratamento}

O American Heart Association recomenda como tratamento inicial e preferencial da DAOP a prática regular de atividade física, principalmente na forma caminhada, com o objetivo de minimizar os efeitos ocasionados pela $\mathrm{Cl}$ na capacidade funcional, qualidade de vida e, quando somados ao tratamento farmacológico, minimizar os fatores de risco cardiovascular. ${ }^{(1,4,22)}$ Se a soma dessas estratégias não forem efetivas e as características clínicas piorarem, deve ser considerado o tratamento cirúrgico no membro obstruído. ${ }^{(4,35,36)}$ 
Contudo, mesmo que o tratamento cirúrgico tenha sido utilizado, a prática de atividade física somadas ao uso de medicamentos para controle de risco cardiovascular, como o tabagismo, diabetes mellitus, dislipidemia e pressão arterial, continuam sendo essenciais. $^{(1,4)}$

\subsection{Exercício como tratamento da doença arterial obstrutiva periférica}

\subsubsection{Exercício como tratamento inicial}

Como mencionado anteriormente, o American Heart Association e American College of Cardiology recomendam o exercício físico supervisionado como tratamento inicial da DAOP. ${ }^{(1,10)}$ Dentre os exercícios recomendados, a caminhada ainda é a mais recomendada para esta população por ser de fácil acesso, não precisar de supervisão de um profissional e, não precisar de aparelho específico para a sua prática. Além disso, a caminha traz diversos benefícios para a capacidade funcional e qualidade de vida de indivíduos com DAOP, ${ }^{(41-44)}$ e em algumas vezes, sendo mais efetivo que o tratamento farmacológico e cirúrgico. ${ }^{(44)}$

Além da caminhada, outras modalidades também têm sido recomendadas e tem demostrado efeitos benéficos na capacidade funcional e qualidade de vida nesta população, como o treinamento de força, ergômetro de braço, subida em escada, exercícios em elípticos e bicicleta ergométrica. ${ }^{(45)}$

\subsubsection{Efeitos do exercício físico na função cardiovascular em pacientes com doença arterial obstrutiva periférica}

Muitos estudos têm evidenciado os efeitos benéficos dos programas de treinamento físico na capacidade funcional em indivíduos com DAOP. Entretanto, os efeitos de diferentes modalidades de exercício em outros parâmetros da saúde não estão bem estabelecidos. Sendo assim, os resultados sobre os efeitos na função cardiovascular são controversos e serão especificados abaixo. 


\subsubsection{Efeitos do exercício físico na pressão arterial}

A maioria dos estudos envolvendo o exercício físico como intervenção em indivíduos com DAOP envolve a pressão arterial braquial. É importante elucidar que cerca de $70 \%$ a $80 \%$ dos pacientes com DAOP apresentam diagnóstico de hipertensão arterial ${ }^{(46,47)}$ e esses valores são importantes para demonstrar um controle de risco cardiovascular, sendo apresentado em diversos estudos. Além disso, para diagnosticar e verificar a gravidade da DAOP, é necessário fazer o exame ITB e a medida de pressão arterial é fundamental para a sua realização. ${ }^{(23)}$

Entretanto, estudos que tem como desfechos principais a pressão arterial e função cardiovascular ainda são controversos. Alguns estudos demonstraram que após os programas de exercício envolvendo o treinamento de força, ergômetro de braço e caminhada reduziram a pressão arterial braquial de pacientes com DAOP. ${ }^{(20,48-}$ 53), enquanto outros não observaram nenhum efeito. ${ }^{(54,55)}$

Pacientes com DAOP apresentam maior pressão arterial central em repouso em comparação com indivíduos sem a doença e essa variável tem se mostrado um forte preditor para risco de eventos cardiovasculares. ${ }^{(56)}$ Embora não exista nenhum estudo investigando os efeitos de um período de programas de exercício na pressão arterial central desses pacientes, um estudo realizado pelo nosso grupo demonstrou que uma única sessão de exercício de força foi capaz de reduzir a pressão arterial central no pós-exercício quando comparado com o grupo controle. ${ }^{(57)}$ Desta forma, futuros estudos são necessários para investigar os possíveis benefícios do exercício físico nesta variável.

\subsubsection{Efeitos do exercício físico na rigidez arterial}

Os efeitos do exercício físico na rigidez arterial de pacientes com DAOP ainda são desconhecidos. Entretanto, um estudo realizado pelo nosso grupo procurou investigar os efeitos de uma única sessão de exercício de força na rigidez arterial. Os resultados demonstraram que não houve alterações na velocidade de onda de pulso carótido-femoral, mas houve alterações positivas nos indicadores de onda refletida (índice de aumento, índice de aumento $75 \mathrm{bpm}$ e pressão de pulso) no pósexercício. ${ }^{(58)}$ 


\subsubsection{Efeitos do exercício físico na modulação autonômica cardíaca}

Alguns estudos demonstraram que após um período de treinamento de exercício de caminha e de força reduziu a frequência cardíaca de repouso. Embora os achados sejam controversos, essas alterações indicam influência positiva dos programas de treinamento na modulação autonômica cardíaca. ${ }^{(20,49,50,59)}$

Apesar dos resultados serem controversos e não terem um consenso, um estudo realizado por Chehuen et al. ${ }^{(50)}$ demonstrou que o exercício de caminhada diminuiu o componente simpático e aumentou o componente parassimpático no coração. Porém, outros estudos não encontraram alterações na variabilidade da frequência cardíaca (indicador da modulação autonômica) após o treinamento com a caminhada. ${ }^{(60,61)}$

\subsubsection{Efeitos do exercício físico no fluxo sanguíneo e na capacidade vasodilatadora}

Pacientes com DAOP apresentam prejuízo endotelial na região afetada devido ao aumento limitado do fluxo sanguíneo em reposta a hiperemia reativa, ocasionando baixa resposta vasodilatadora. ${ }^{(33)}$ É importante ressaltar que a baixa resposta vasodilatadora está associada com alto risco de evento cardiovascular em pacientes com DAOP. ${ }^{(62)}$

Um estudo de revisão demonstrou que após um período de exercício parece não alterar o fluxo sanguíneo na musculatura da panturrilha durante o repouso. ${ }^{(62)}$ Entretanto, a capacidade vasodilatadora desses pacientes parece melhorar após um período de intervenção com exercício. ${ }^{(63,64)}$

\subsection{Nível de atividade física de pacientes com doença arterial obstrutiva periférica}

A prática de atividade física tem sido recomendada para toda a população, inclusive pacientes com DAOP, que devem praticar pelo menos 150 minutos de atividade física moderada à vigorosa por semana. ${ }^{(65)}$ Entretanto, um estudo recente realizado pelo nosso grupo demonstrou que pacientes com DAOP com 
sintomas de $\mathrm{Cl}$ despenderam a maior parte do seu tempo em comportamento sedentário e apenas $3,4 \%$ dos pacientes foram considerados ativos fisicamente de acordo com as recomendações atuais de atividade física. ${ }^{(30)}$ Além disso, também foi observado que nenhum paciente com idade acima de 70 anos atingiu as recomendações de atividade física. ${ }^{(30)}$

Nesse sentido, um estudo realizado por Barbosa et al. ${ }^{(66)}$ demonstrou que as principais barreiras para a não prática de atividade física de pacientes com DAOP estão relacionadas com os sintomas da doença. A dor induzida pelo exercício (75\%), a necessidade de descansar por causa da dor (65\%) e o cansaço $(59 \%)$ foram as principais barreiras pessoais relatadas pelos pacientes. ${ }^{(66)}$ Também foram citadas as barreiras ambientais como ter osbstáculos nas ruas que agravam a dor na perna (75\%) e calçadas insatisfatórias (64\%). ${ }^{(66)}$

Além disso, tem sido demonstrado que menores níveis de atividade física está relacionado com um maior risco de mortalidade por evento cardiovascular. ${ }^{(67)}$ Um estudo realizado por Garg et al. ${ }^{(68)}$ demonstrou que pacientes com DAOP com níveis mais baixos de atividade física durante a vida diária foram associados a maior risco de mortalidade por evento cardiovascular em comparação com pacientes que tinham níveis mais altos de atividade física. ${ }^{(68)}$

Dessa forma, programas de atividade física são necessários para contornar as barreiras que impactam a prática de atividade física dessa população para auxiliar no aumento de seus níveis de atividade física diária e, principalmente, que tenham um efeito positivo na saúde cardiovascular em pacientes com DAOP. 


\section{MÉTODOS}

\subsection{Caracterização do estudo}

Trata-se de um estudo observacional longitudinal. Este projeto faz parte de um estudo maior que foi iniciado no ano de 2015 e foi aprovado pelo comitê de ética em pesquisa do Hospital Israelita Albert Einstein (CAAE: 42379015.3.0000.0071) e do Hospital das Clínicas da Faculdade de Medicina da Universidade de São Paulo (CAAE: 42379015,3,3002,0068). Na primeira fase do estudo, 303 pacientes foram recrutados e submetidos a avaliação do nível de atividade física, pressão arterial clínica, pressão arterial central, rigidez arterial e, modulação autonômica da frequência cardíaca. Nesta segunda etapa, após 2 anos, todos os pacientes incluídos na primeira fase foram convidados para serem submetidos novamente a todos os exames supracitados. O delineamento experimental do estudo está apresentado na figura 3.

\begin{tabular}{|c|c|}
\hline Primeira Fase & Segunda Fase \\
\hline Nível de Atividade Física & Nível de Atividade Física \\
\hline Pressão Arterial & Pressão Arterial \\
\hline Rigidez Arterial & Rigidez Arterial \\
\hline Variabilidade de Frequência Cardíaca & Variabilidade de Frequência Cardíaca \\
\hline
\end{tabular}

Figura 3. Desenho experimental do estudo

\subsection{Recrutamento, triagem e dimensionamento da amostra}

O recrutamento dos pacientes foi realizado no Ambulatório de Claudicação Intermitente $(\mathrm{Cl})$ do Hospital Israelita Albert Einstein e no Ambulatório de Cl do Hospital das Clínicas da Faculdade de Medicina da Universidade de São Paulo.

$\mathrm{Na}$ fase 1, os critérios de inclusão ao estudo foram: idade igual ou maior a 45 anos, de ambos os sexos, índice tornozelo-braço <0,90 em um ou ambos os membros e apresentar sintomas de Cl. Aqueles pacientes que não tiveram o ITB identificado não foram incluídos do presente estudo. Previamente á coleta de dados os 
pacientes foram informados sobre os procedimentos envolvidos na realização do estudo e assinaram o Termo de Consentimento Livre e Esclarecido.

\subsection{Medidas e avaliações}

Os pacientes que aceitaram participar da fase 2 do estudo foram submetidas as mesmas avaliações da fase $1 \mathrm{em}$ duas visitas distintas. Na primeira visita foram novamente obtidos os dados clínicos e sócio-demográficos, e os pacientes receberam o monitor de atividade física. Na segunda visita, foram obtidas as medidas de pressão arterial clínica, pressão arterial central, modulação anatômica cardíaca e a rigidez arterial. Para tanto, os pacientes foram orientados a seguir as seguintes recomendações: realizar uma refeição leve, não realizar exercício físico pelo menos 24 horas antes, leve, não ingerir bebidas alcoólicas e cafeínadas, não fumar 12 horas antes da sessão e manter a rotina normal de utilização da medicação.

\subsection{Variável independente - nível de atividade física}

O nível de atividade física foi obtido a partir de acelerometria, sendo utilizados equipamentos da marca Actigraph, modelo GT3X+, Estados Unidos. Cada paciente foi instruído a utilizar o acelerômetro durante sete dias consecutivos, retirando-o apenas para dormir, tomar banho ou para realizar atividades aquáticas. $O$ equipamento foi acoplado a um cinto elástico e fixado no lado direito do quadril. Para fins de análise, foram considerados como dados válidos um mínimo de 10 horas de gravações de atividade diária, durante pelo menos quatro dias, sendo três dias da semana e um dia do final de semana. Para classificação da intensidade do nível de atividade física, foram estabelecidos limiares (pontos de corte) e faixas classificatórias de acordo com os counts obtidos, sendo que foram considerados como atividade física total (100-1951 $\left.\mathrm{min}^{-1}\right)$. Levando em consideração a linha de base, os sujeitos foram classificados em tercis de acordo com os deltas (atividade física total fase 2 - atividade física total fase 1) como reduziu muito, reduziu pouco e aumentou de acordo com o nível de atividade física total como demonstrado na figura 4. 


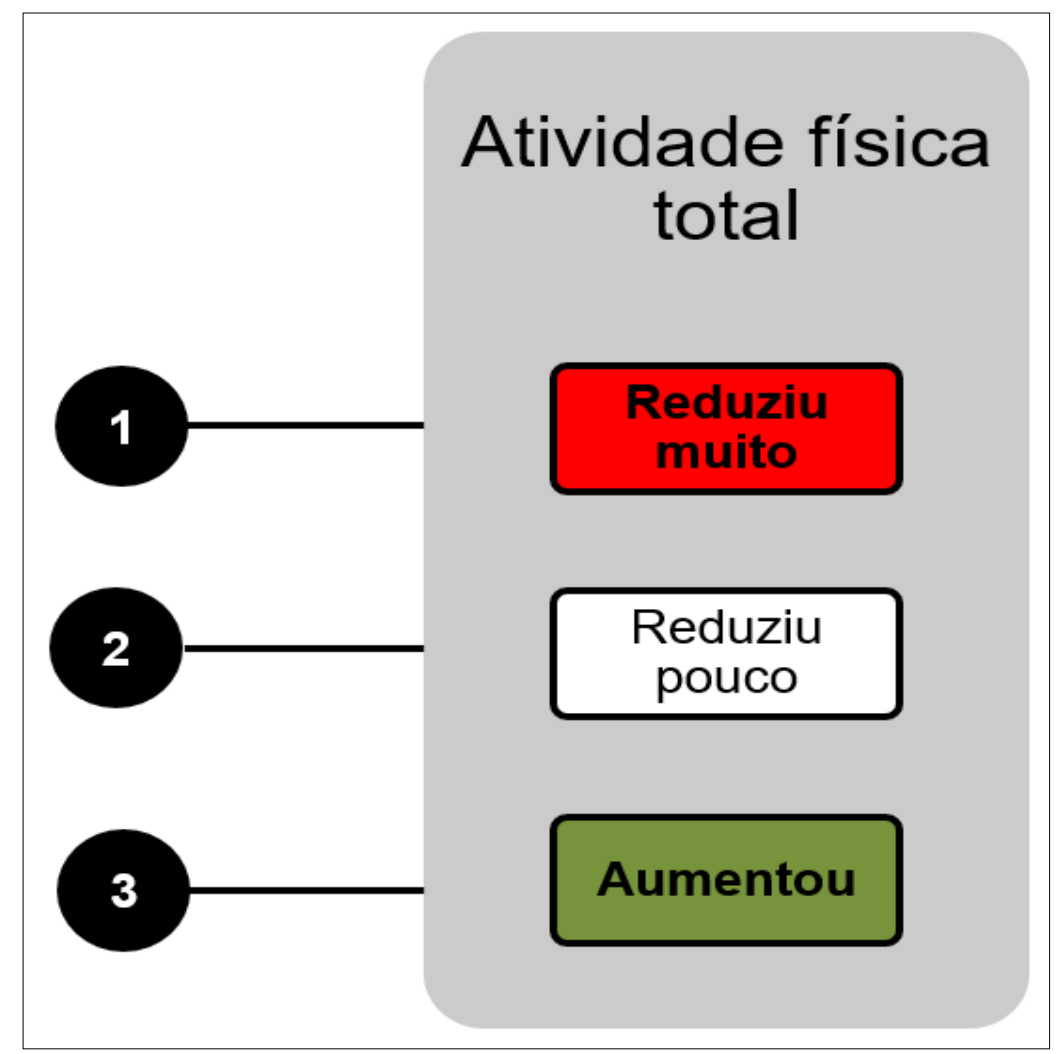

Figura 4. Tercis de acordo com a atividade física total

\subsection{Desfechos primários - indicadores de risco cardiovascular}

\subsubsection{Pressão arterial clínica}

A pressão arterial clínica dos pacientes foi avaliada por meio de um monitor (HEM-742, Omron Healthcare, Japão), o qual consiste em um aparelho eletrônico e digital da medida da pressão arterial de braço, com deflação e inflação automática de ar. Para essa medida, os pacientes permaneceram em repouso por dez minutos na posição sentada. Foram realizadas três medidas consecutivas, com um minuto de intervalo, em ambos os braços e com tamanho do manguito adequado para a circunferência do braço. O valor utilizado foi a média das três medidas, conforme recomendado pela Sociedade Brasileira de Cardiologia. ${ }^{(69)}$ Esta medida sempre foi realizada pelo mesmo avaliador experiente no mesmo paciente. 


\subsubsection{Pressão arterial central}

A medida da pressão arterial central foi realizada pela análise da onda de pulso da artéria radial por meio da técnica de tonometria de aplanação (SphymoCor, AtCor Medical, Australia). Para tanto, após pelo menos 15 minutos de repouso na posição supina, foram utilizados 11 segundos de gravação da onda de pressão arterial radial. Após esse procedimento o software SphygmoCor ${ }^{\circledR}$, deriva a onda de pressão da aorta ascendente, equivalente à onda de pressão medida por um cateter invasivo obtendo-se os valores de pressão arterial central sistólica e diastólica. ${ }^{(70)}$ Para melhor acurácia na medida, somente foram considerados válidos, os valores cujos índices foram maiores que $90 \%$.

\subsubsection{Modulação autonômica do sistema cardiovascular}

A modulação autonômica do sistema cardiovascular foi avaliada pela técnica da variabilidade da frequência cardíaca. Para tanto, os pacientes permaneceram em repouso deitados por 15 minutos, em seguida, durante o mesmo período foram registrados os intervalos RR por meio de um monitor de frequência cardíaca válido para esta função (Polar V800, Polar Electro, Finlândia). Entretanto, os primeiros cinco minutos foram excluídos, e, foi considerado um sinal válido aqueles com pelos menos 10 minutos de sinal estacionário.

Após a coleta, os intervalos RR foram exportados para o programa Kubios HRV (Biosignal Analysis and Medical Imaging Group, Finlândia), cujas análises foram realizadas pelo domínio do tempo e da frequência. Os parâmetros do domínio do tempo, desvio padrão de todos os intervalos RR (SDNN), raiz quadrada da média do quadrado das diferenças entre os intervalos RR normais adjacentes (RMSSD) e porcentagem dos intervalos adjacentes com mais de $50 \mathrm{~ms}$ (PNN50) foram obtidos. ${ }^{(71)}$ Os parâmetros do domínio da frequência foram obtidos pela técnica da análise espectral por meio do método autorregressivo. Foram consideradas como fisiologicamente significativas as frequências entre 0,04 e $0,4 \mathrm{~Hz}$, sendo o componente de baixa frequência representado pelas oscilações entre 0,04 e $0,15 \mathrm{~Hz}$ e o componente de alta frequência entre 0,15 e $0,4 \mathrm{~Hz}$. O poder de cada componente espectral foi calculado em termos normalizado(s), que foi realizada dividindo-se o poder 
de cada banda pelo poder total, do qual foi subtraído o valor de banda de muito baixa frequência $(<0,04 \mathrm{~Hz})$, sendo o resultado multiplicado por $100{ }^{(71)}$

\subsubsection{Rigidez arterial}

A estimativa da rigidez arterial foi realizada a partir da avaliação da velocidade da onda de pulso aórtico carótido-femoral. Para tanto, foi utilizada a técnica de tonometria de aplanação. As ondas de pulso aórtico carótido-femoral foram registradas sequencialmente por transdutores transcutâneos, posicionados acima das artérias carótida e femoral direita, utilizando-se um aparelho de tonometria por aplanação (SphygmoCor, AtCor Medical, Austrália). O registro eletrocardiográfico foi obtido simultaneamente às medidas de onda de pulso aórtico carótido-femoral como padrão de referência para calcular o tempo de trânsito da onda. Duas distâncias de superfícies foram medidas pelo investigador: uma entre o ponto de gravação na artéria carótida e da fúrcula esternal (distância 1) e a outra entre a fúrcula esternal e o ponto de gravação em a artéria femoral (distância 2). A distância percorrida pela onda de pulso foi calculada como "distância 2" - "distância 1". A onda de pulso aórtico carótidofemoral foi calculada como: onda de pulso aórtico carótido-femoral = distância percorrida pela onda de pulso 1/4 (m)/tempo de trânsito(s).

\subsection{Análise estatística}

As estatísticas descritivas para variáveis contínuas foram expressas como média, desvio-padrão e 95\% de intervalo de confiança (IC 95\%). As variáveis categóricas foram apresentadas como percentual e seu IC de 95\%. As comparações de médias ao longo do tempo foram analisadas por meio na análise de variância [ANOVA] para medidas repetidas com o teste post-hoc de Bonferroni. O nível de significância adotado foi $5 \%$ e as análises foram realizadas com o auxílio do programa estatístico IBM Statistical Package for Social Sciences (SPSS) 25. 
4 RESULTADOS

O período de recrutamento para o retorno dos pacientes do presente estudo ocorreu entre janeiro de 2017 a julho de 2019. Durante esse período, 96 pacientes aceitaram participar novamente e destes, 19 pacientes foram considerados não elegíveis por não apresentarem dados dos níveis de atividade física. Portanto, a amostra final desse estudo compreende dados de 77 pacientes com DAOP. O fluxograma do estudo está demonstrado na figura 5.
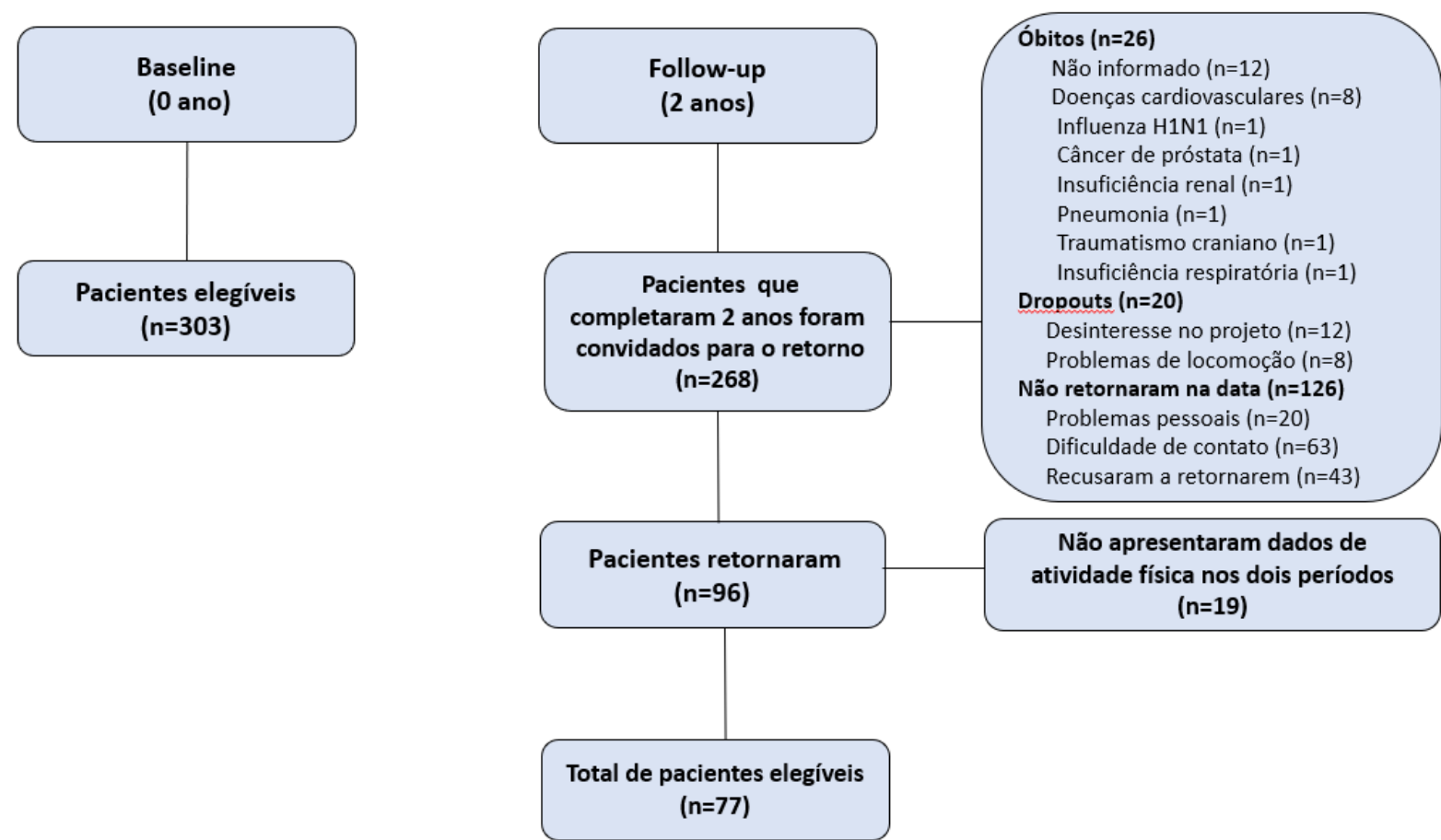

Figura 5. Fluxograma dos pacientes envolvidos no estudo

A tabela 1 apresenta os dados do tempo despendido em atividade física total no baseline e follow-up.

Tabela 1. Nível de atividade física de pacientes no baseline e follow-up

\begin{tabular}{lccccc}
\hline Variáveis & $\mathbf{n}$ & Baseline & $\mathbf{n}$ & Follow-up & Valor-p \\
\hline Atividade física total & 77 & $2257,6 \pm 774,5$ & 77 & $2041 \pm 676,2$ & $<0,01$ \\
(minutos/semana) & 77 & 7,8 & 77 & 3,9 & 0,08 \\
Atingiram as recomendações (\%) & 77
\end{tabular}

Dados apresentados como média \pm desvio padrão ou frequência relativa. 
Podemos observar na tabela 1 que os valores do nível de atividade física total se modificaram no momento follow-up, havendo diferenças significativas em relação ao baseline. Apesar de não apresentar significância estatística, É importante destacar que houve tendência de redução na \% de pacientes que atingem as recomendações de atividade física $(7,8 \%$ no baseline versus $3,9 \%$ no follow-up, $p=0,08)$.

A tabela 2 apresenta as características clínicas gerais e fatores de risco dos 77 pacientes no baseline e follow-up (2 anos).

Tabela 2. Características clínicas e fatores de risco de pacientes com claudicação intermitente no baseline e follow-up

\begin{tabular}{lccc}
\hline Variáveis & $\begin{array}{c}\text { Valores } \\
\text { Baseline } \\
(\mathbf{n}=\mathbf{7 7})\end{array}$ & $\begin{array}{c}\text { Valores } \\
\text { Follow-up } \\
(\mathbf{n = 7 7 )}\end{array}$ & Valor-p \\
\hline Idade (anos) & $65,5 \pm 9,0$ & $67,7 \pm 9,0$ & $<0,01$ \\
Peso (kg) & $74,3 \pm 14,1$ & $73,4 \pm 13,5$ & 0,16 \\
Altura (metros) & $1,64 \pm 0,1$ & $1,63 \pm 0,1$ & 0,22 \\
Índice de massa corporal $\left(\mathrm{kg} / \mathrm{m}^{2}\right)$ & $27,5 \pm 4,7$ & $27,3 \pm 4,3$ & 0,46 \\
Índice tornozelo-braço & $0,61 \pm 0,19$ & $0,52 \pm 0,19$ & $<0,01$ \\
Distância total de caminhada de 6 minutos & $346,6 \pm 86,2$ & $361,4 \pm 103,2$ & 0,23 \\
(metros) & & & \\
Fatores de risco & 42,7 & 47,4 & 0,18 \\
$\quad$ Diabetes mellitus, \% & 82,7 & 85,7 & 0,15 \\
$\quad$ Hipertensão, \% & 84 & 90,9 & 0,02 \\
Dislipidemia, \% & 26,7 & 40 & 0,12 \\
Obesidade, \% & 34,2 & 39,5 & 0,39 \\
Doença arterial coronariana, \% & 14,9 & 20,8 & 0,04 \\
Acidente vascular cerebral, \% & 12,5 & 15,3 & 0,44 \\
Insuficiência Cardíaca Congestiva \% & 12,5 & 12 & 0,65 \\
$\quad$ Câncer, \% & & &
\end{tabular}

Dados apresentados como média \pm desvio padrão ou frequência relativa.

No início do estudo, a amostra foi composta predominantemente por pacientes idosos, com sobrepeso e, com diversos fatores de risco, dentre eles podemos destacar a hipertensão arterial e a dislipidemia. É interessante notar que após o período de dois anos, houve redução significante do índice tornozelo-braço e aumento da prevalência de dislipidemia e acidente vascular cerebral $(<0,05)$. Nas demais características clínicas e fatores de risco, não observamos mudanças no período de follow-up.

A tabela 3 apresenta os valores da frequência cardíaca e pressão arterial entre baseline e follow-up. 
Tabela 3. Pressão arterial e frequência cardíaca dos pacientes no baseline e follow-up

\begin{tabular}{lccccc}
\hline Variáveis & $\mathbf{n}$ & Baseline & $\mathbf{n}$ & Follow-up & Valor-p \\
\hline Frequência Cardíaca em repouso & 72 & $64,4 \pm 11,5$ & 72 & $67,7 \pm 17,2$ & 0,12 \\
Pressão Arterial Sistólica (mmHg) & 73 & $133,3 \pm 21,0$ & 73 & $132,5 \pm 21,0$ & 0,69 \\
Pressão Arterial Diastólica (mmHg) & 73 & $73,0 \pm 10,2$ & 73 & $72,7 \pm 10,6$ & 0,74 \\
$\begin{array}{l}\text { Pressão Arterial Sistólica central } \\
\text { (mmHg) }\end{array}$ & 62 & $130,9 \pm 22,3$ & 62 & $128,0 \pm 21,4$ & 0,43 \\
$\begin{array}{l}\text { Pressão Arterial Diastólica central } \\
\text { (mmHg) }\end{array}$ & 62 & $75,2 \pm 9,9$ & 62 & $74,6 \pm 9,8$ & 0,79 \\
\hline
\end{tabular}

Dados apresentados como média \pm desvio padrão.

No baseline os pacientes apresentaram níveis de pressão arterial entre pré-hipertensão e hipertensão. Não houve alterações significantes na frequência cardíaca e pressão arterial entre o período baseline e follow-up (todos com p $>0,05$ ). A tabela 4 apresenta os dados de rigidez arterial entre o baseline e follow-up.

Tabela 4. Indicadores de rigidez arterial e onda refletida de pacientes no baseline e follow-up

\begin{tabular}{lccccc}
\hline Variáveis & $\mathbf{n}$ & Baseline & $\mathbf{n}$ & Follow-up & Valor-p \\
\hline Pressão de Pulso (mmHg) & 62 & $55,7 \pm 18,2$ & 62 & $52,5 \pm 18,3$ & 0,09 \\
Índice de Aumento (\%) & 60 & $32,3 \pm 11,1$ & 60 & $30,6 \pm 13,2$ & 0,59 \\
Índice de Aumento 75bpm (\%) & 60 & $26,6 \pm 9,6$ & 60 & $26,9 \pm 10,6$ & 0,42 \\
Velocidade de Onda de Pulso (m/s) & 43 & $9,0 \pm 2,9$ & 43 & $10,6 \pm 3,3$ & $<0,01$ \\
\hline \multicolumn{7}{l}{ Dados apresentados como média \pm desvio padrão. }
\end{tabular}

Podemos observar na tabela 4 que tivemos uma perda amostral na pressão de pulso de 15 pacientes, índice de aumento e índice de aumento corrigido por $75 \mathrm{bpm}$ de 17 pacientes e velocidade de onda de pulso de 34 pacientes devido a presença de arritmias ou pulsação fraca da artéria radial e/ou femoral, o que impossibilitou a análise dos dados. Não houve alterações dos indicadores de rigidez arterial entre o período baseline e follow-up, exceto para a velocidade de onda de pulso, no qual foi observado aumento dos seus valores no período de follow-up em relação ao baseline (baseline $=9,0 \pm 2,9$ versus follow-up $=10,6 \pm 3,3, p=0,003$ ).

A tabela 5 apresenta as variáveis da modulação autonômica cardíaca no baseline e follow-up. 
Tabela 5. Variáveis de análise da modulação autonômica cardíaca no baseline e followup

\begin{tabular}{lccccc}
\hline Variáveis & n & Baseline & n & Follow-up & Valor-p \\
\hline Domínio do tempo & & & & & \\
SDNN (ms) & 39 & $45,6 \pm 31,4$ & 39 & $24,3 \pm 13,3$ & $<0,01$ \\
RMSSD (ms) & 39 & $40,6 \pm 49,1$ & 39 & $24,7 \pm 16,0$ & 0,18 \\
pNN50 (\%) & 39 & $11,3 \pm 17,4$ & 39 & $7,8 \pm 12,2$ & 0,23 \\
Domínio da frequência & & & & & \\
Baixa frequência (un) & 39 & $56,8 \pm 22,3$ & 39 & $57,9 \pm 16,8$ & 0,97 \\
Alta frequência (un) & 39 & $43,0 \pm 22,2$ & 39 & $41,9 \pm 16,7$ & 0,98 \\
Razão BF/AF (un) & 39 & $2,2 \pm 2,1$ & 39 & $1,7 \pm 1,1$ & 0,69 \\
\hline
\end{tabular}

BF/AF: balanço simpato-vagal; pNN50: percentual dos intervalos adjacentes com mais de $50 \mathrm{~ms}$; RMSSD: raiz quadrada da média do quadrado das diferenças entre os intervalos RR normais adjacentes; SDNN: desvio padrão de todos os intervalos RR.

Podemos observar na tabela 5 que não foram apresentados dados de 38 pacientes devido a presença de arritmias e marcapasso, e esses dados foram considerados não elegíveis para a análise. Os valores da modulação autonômica cardíaca não se modificaram entre baseline e follow-up, exceto para o SDNN (ms) que apresentou valores menores, havendo diferenças significativas no follow-up em comparação ao baseline. A modulação simpática, analisados pela banda de baixa frequência e razão $B F / L F$ estavam aumentados no início do estudo se mantiveram no momento de follow-up.

A tabela 6 apresenta dados das características clínicas gerais e fatores de risco de pacientes de acordo com os deltas dos tercis despendido em atividade física total (reduziu muito $=\Delta-788,5 \pm 510,7 \mathrm{~min} /$ semana; reduziu pouco $=\Delta$ $163,8 \pm 115,4 \mathrm{~min} /$ semana; aumentou $=\Delta 281,8 \pm 182,4 \mathrm{~min} /$ semana $)$.

Tabela 6. Características clínicas e fatores de risco de acordo com os deltas dos tercis do tempo despendido em atividade física total

\begin{tabular}{lcccc}
\hline Variáveis & $\begin{array}{c}\text { Reduziu } \\
\text { muito } \\
(\mathbf{n = 2 5 )}\end{array}$ & $\begin{array}{c}\text { Reduziu } \\
\text { pouco } \\
(\mathbf{n = 2 6 )}\end{array}$ & $\begin{array}{c}\text { Aumentou } \\
(\mathbf{n = 2 6 )}\end{array}$ & Valor-p \\
\hline Idade (anos) & $64,8 \pm 9,7$ & $64,7 \pm 8,6$ & $67,1 \pm 8,8$ & 0,57 \\
Peso (kg) & $73,1 \pm 12,4$ & $77,8 \pm 15,6$ & $72,1 \pm 14,1$ & 0,31 \\
Altura (metros) & $1,62 \pm 0,07$ & $1,65 \pm 0,08$ & $1,6 \pm 0,09$ & 0,66 \\
Índice de Massa Corporal (kg/m²) & $27,6 \pm 5,4$ & $28,4 \pm 4,7$ & $26,5 \pm 3,8$ & 0,35 \\
Índice tornozelo-braço & $0,65 \pm 0,16$ & $0,59 \pm 0,21$ & $0,59 \pm 0,21$ & 0,42 \\
Distância total de caminhada de 6 & $335,4 \pm 96,8$ & $367,1 \pm 80,6$ & $334,9 \pm 78,9$ & 0,32 \\
minutos (metros) & & & & \\
Fatores de risco & 20,8 & $53,8^{*}$ & $52^{*}$ & 0,03 \\
$\quad$ Diabetes mellitus, \% & 79,2 & 88,5 & 80 & 0,62 \\
$\quad$ Hipertensão, \% & 87,5 & 84,6 & 80 & 0,77 \\
$\quad$ Dislipidemia, \% & 25 & 44,4 & 13,6 & 0,89 \\
$\quad$ Obesidade, \% & & & & continua $\ldots$
\end{tabular}




\begin{tabular}{lcccc}
...continuação & 20,8 & 45,8 & 36 & 0,18 \\
\hline Doença arterial coronariana, \% & 12,5 & 16 & 16 & 0,92 \\
Acidente Vascular Cerebral, \% & 8,3 & 8,7 & 20 & 0,37 \\
Insuficiência Cardíaca Congestiva, \% & 9,1 & 12 & 16 & 0,77 \\
Câncer, \% & .
\end{tabular}

Dados apresentados como média \pm desvio padrão. * diferente do grupo 1; - Diferente do grupo 2.

Podemos observar na tabela 6 que não houve diferenças significativas das características clínicas entre os grupos no baseline. Os fatores de risco dos pacientes não apresentaram diferenças significativas entre os grupos no baseline, apenas o grupo que reduziu muito apresentou menor prevalência do diabetes mellitus em relação ao grupo que reduziu pouco e aumentou muito.

A tabela 7 apresenta as alterações das variáveis cardiovasculares de pacientes de acordo com os tercis do tempo despendido em atividade física total (reduziu muito $=\Delta-788,5 \pm 510,7 \mathrm{~min} / \mathrm{semana} ;$ reduziu pouco $=\Delta-$ $163,8 \pm 115,4 \mathrm{~min} /$ semana; aumentou $=\Delta 281,8 \pm 182,4 \mathrm{~min} /$ semana).

Tabela 7. Alterações $(\Delta)$ nas variáveis cardiovasculares de acordo com as alterações na atividade física total

\begin{tabular}{lcccc}
\hline Variáveis & $\begin{array}{c}\text { Reduziu } \\
\text { muito } \\
(\mathbf{n = 2 5 )}\end{array}$ & $\begin{array}{c}\text { Reduziu } \\
\text { pouco } \\
(\mathbf{n = 2 6 )}\end{array}$ & $\begin{array}{c}\text { Aumentou } \\
(\mathbf{n = 2 6 )}\end{array}$ & Valor-p \\
\hline$\Delta$ Frequência Cardíaca (bpm) & $2,5 \pm 19,7$ & $4,1 \pm 14,4$ & $3,15 \pm 13,6$ & 0,94 \\
$\Delta$ Pressão Arterial Sistólica (mmHg) & $1,4 \pm 17,7$ & $-5,8 \pm 21,8$ & $0,4 \pm 20,6$ & 0,40 \\
$\Delta$ Pressão Arterial Diastólica (mmHg) & $0,6 \pm 7,7$ & $-3,2 \pm 9,7$ & $1,7 \pm 10,9$ & 0,17 \\
$\Delta$ Pressão Arterial Sistólica Central (mmHg) & $-0,2 \pm 23,7$ & $-5,0 \pm 23,9$ & $-3,1 \pm 22,1$ & 0,82 \\
$\Delta$ Pressão Arterial Diastólica Central (mmHg) & $1,2 \pm 10,0$ & $-1,7 \pm 11,0$ & $-0,8 \pm 9,8$ & 0,66 \\
$\Delta$ Pressão de Pulso aórtica (mmHg) & $-1,2 \pm 16,4$ & $-3,0 \pm 21,8$ & $-5,0 \pm 14,5$ & 0,79 \\
$\Delta$ Índice de Aumento (\%) & $-1,0 \pm 12,2$ & $-2,1 \pm 114,6$ & $-1,9 \pm 11,6$ & 0,96 \\
$\Delta$ Índice de Aumento 75bpm (\%) & $-0,7 \pm 8,6$ & $0,5 \pm 8,9$ & $0,8 \pm 8,4$ & 0,83 \\
$\Delta$ Velocidade de onda de pulso (m/s) & $0,5 \pm 2,3$ & $2,2 \pm 3,2$ & $1,9 \pm 3,7$ & 0,32 \\
$\Delta$ SDNN (m/s) & $-20,2 \pm 23,2$ & $-35,3 \pm 42,5$ & $-4,9 \pm 20,4$ & 0,58 \\
$\Delta$ RMSSD (m/s) & $-9,2 \pm 38,0$ & $-36,9 \pm 70,0$ & $5,7 \pm 16,6$ & 0,84 \\
$\Delta$ pNN50 (\%) & $-3,4 \pm 17,6$ & $-7,7 \pm 21,3$ & $1,5 \pm 6,3$ & 0,37 \\
$\Delta$ Baixa frequência (\%) & $1,1 \pm 23,8$ & $5,9 \pm 25,0$ & $-5,8 \pm 20,2$ & 0,47 \\
$\Delta$ Alta frequência (\%) & $-1,3 \pm 23,6$ & $-5,6 \pm 25,0$ & $0,7 \pm 24,2$ & 0,77 \\
$\Delta$ BF/AF & $-0,2 \pm 1,9$ & $-0,5 \pm 2,8$ & $-0,7 \pm 1,9$ & 0,86 \\
\hline BF/AF: bancon
\end{tabular}

BF/AF: balanço simpato-vagal; pNN50: percentual dos intervalos adjacentes com mais de 50ms; RMSSD: raiz quadrada da média do quadrado das diferenças entre os intervalos RR normais adjacentes; SDNN: desvio padrão de todos os intervalos RR. Dados apresentados como média \pm desvio padrão. * diferente do grupo $1 ; \square$ Diferente do grupo 2. 
Podemos observar na tabela 7 que não houve diferenças significativas entre os deltas das variáveis cardiovasculares nos tercis das alterações do tempo despendido em atividade física total. 


\section{DISCUSSÃO}

Os principais achados deste estudo foram: a) Houve redução do tempo despendido em atividade física total no seguimento de 2 anos em pacientes com DAOP; b) Alterações do tempo despendido em atividade física total não foram associados com as alterações nos indicadores de risco cardiovascular no seguimento de 2 anos. Desta forma, nossos dados sugerem que a atividade física total parece não ser capaz de promover modificações nos indicadores de risco cardiovascular de pacientes com DAOP no seguimento de 2 anos.

\subsection{Nível de atividade física no baseline e follow-up de 2 anos}

As recomendações atuais de atividade física para a população em geral, incluindo pacientes com DAOP, consistem em praticar pelos menos 150 minutos de atividade física moderada ou 75 minutos de atividades físicas vigorosas e/ou uma combinação equivalente de atividades físicas moderadas a vigorosas por semana. ${ }^{(65)}$ No presente estudo, observamos que pacientes com DAOP apresentaram baixos níveis de atividade física e despenderam a maior parte do seu tempo em comportamento sedentário. De fato, no baseline apenas $7,8 \%$ dos nossos pacientes atingiram as recomendações para a prática de atividade física e, após 2 anos, apenas $3,9 \%$ atingiram as recomendações. Esses valores são semelhantes ao encontrado em um estudo anterior realizado pelo nosso grupo em que apenas 3,4\% dos indivíduos com DAOP atingiram as recomendações de atividade física. ${ }^{\left({ }^{30}\right)}$ Esses resultados são alarmantes uma vez que, as principais diretrizes sobre DAOP são claras em recomendar como forma inicial do tratamento clínico, a prática regular de atividade física. Assim, o achado de nosso estudo de que a maioria dos pacientes com DAOP não modificam e, até pioram seus níveis de atividade física, destacam a necessidade de explorar novas estratégias para aumentar a atividade física nesses pacientes. ${ }^{(72,73)}$

\subsection{Modificações nas características clínicas no baseline e follow-up de 2 anos}

No presente estudo, os pacientes incluídos tiveram características semelhantes à de estudos anteriores do nosso grupo, sendo, em sua maioria, idosos e 
com sobrepeso. ${ }^{(49)}$ Os pacientes apresentaram no início do estudo severidade moderada da DAOP, sendo que, ao longo do seguimento de 2 anos, observamos redução dos valores de ITB $(0,61 \pm 0,19$ versus $0,52 \pm 0,19$, no baseline e follow-up, respectivamente). A redução do ITB não é um resultado surpreendente, uma vez que estudos anteriores demonstram redução dos seus valores ao longo do tempo. ${ }^{(74)}$

Além disso, a maioria dos pacientes apresentaram fatores de risco associados com a DAOP como hipertensão arterial $(85,7 \%)$, dislipidemia $(90,9 \%)$, diabetes mellitus $(47,4 \%)$ e doença arterial coronariana (39,5\%). Adicionalmente, houve aumento da incidência de acidente vascular cerebral (14,9\% e 20,8\% no baseline e follow-up, respectivamente) e dislipidemia (84\% e 90,9\% no baseline e follow-up, respectivamente) no segmento de 2 anos. De fato, como a DAOP é uma doença sistêmica, é esperado que esses pacientes possam sofrer eventos fatais e não fatais decorrentes de processos ateroscleróticos em outros leitos arteriais. ${ }^{(1,3)}$

\subsection{Modificações nos indicadores de risco cardiovascular no baseline e follow- up de 2 anos}

A pressão arterial é um forte preditor para doenças cardiovasculares. Níveis reduzidos de pressão arterial estão associados em uma redução de $6 \%$ de mortalidade por acidente vascular cerebral e uma diminuição de 4\% na mortalidade por doença cardíaca coronariana. ${ }^{(75)}$ No estudo em discussão, os pacientes apresentaram média de $133 \pm 21 \mathrm{mmHg}$ e 132,5 $\pm 21,0 \mathrm{mmHg}$ de pressão arterial sistólica no baseline e follow-up, respectivamente, não apresentando modificações no período de seguimento de 2 anos. Desta forma, os resultados sugerem que os pacientes apresentaram controle adequado da pressão arterial, que podem ser explicados, em parte, pela possível manutenção da utilização dos medicamentos anti-hipertensivos ao longo do estudo.

Observando os indicadores de rigidez arterial dos pacientes, representados pela velocidade de onde de pulso, observamos um aumento significativo ao longo dos 2 anos $(9,0 \pm 2,9$ e 10,6 \pm 3,3ms, no baseline e follow-up, respectivamente), indicando uma piora na rigidez arterial no seguimento de 2 anos. A rigidez arterial está associada com a progressão da aterosclerose e, com o seu aumento, é um forte marcador de risco de mortalidade cardiovascular e eventos 
cardiovasculares. ${ }^{(76,77)}$ Além disso, a idade tem um papel importante na rigidez arterial. Um estudo recente realizado pelo nosso grupo demonstrou forte associação entre a rigidez arterial e a idade em pacientes com DAOP. ${ }^{(78)}$ Por outro lado, a rigidez arterial periférica, representados pela pressão de pulso, índice de aumento e índice de aumento corrigido por $75 \mathrm{bpm}$ não apresentaram alterações significativas entre os baseline e o follow-up no seguimento de 2 anos. Uma possível explicação para isso é que a onda refletida tem relação com a pressão arterial diastólica, sendo que esta variável não sofreu alterações ao longo do estudo. ${ }^{(58)}$

A variabilidade da frequência cardíaca tem sido outra ferramenta clínica importante para indicação de risco cardiovascular em diversas populações, ${ }^{(79)}$ sendo que indivíduos com DAOP apresentam disfunção autonômica, aumentando as chances de doenças e mortalidade por doença cardiovascular. ${ }^{(80)}$ Os pacientes do presente estudo apresentaram valores da variabilidade da frequência cardíaca (SDNN, RMSSD, pNN50, alta frequência, baixa frequência e relação baixa e alta frequência) similares a outro estudo com pacientes com DAOP. ${ }^{(61)}$ De fato, os valores da variabilidade da frequência cardíaca em repouso indicam que os pacientes do presente estudo apresentam maior modulação do sistema simpático e menor modulação do sistema parassimpático. No seguimento de 2 anos, apenas o indicador SDNN apresentou redução $(45,6 \pm 31,4$ e $24,3 \pm 13,3$ do baseline para o follow-up), o que parece sugerir que esse indicador pode ser mais sensível às alterações na modulação autonômica cardíaca ao longo do tempo.

\subsection{Influência do nível de atividade física sobre os indicadores de risco cardiovascular durante o período de 2 anos de seguimento}

Esse é o primeiro estudo longitudinal que buscou verificar a influência do nível de atividade física total sobre os indicadores de risco cardiovascular de pacientes com DAOP. A hipótese era de que aumento do tempo despendido em atividade física total estaria associado a melhoria nos indicadores de saúde cardiovascular em comparação aos seus pares menos ativos durante o seguimento do estudo. No entanto, essa hipótese não foi confirmada.

Estudos transversais têm indicado que maior tempo despendido em atividade física leve e moderado à vigoroso estão associados a menores valores de 
pressão arterial braquial, pressão arterial central e rigidez arterial. ${ }^{\left({ }^{81-84)}\right.}$ Além disso, maiores níveis de atividade física também foram associados a maiores valores da variabilidade da frequência cardíaca. ${ }^{\left({ }^{85}\right)}$ Esses fatores são muito importantes, uma vez que melhores valores dos indicadores de risco cardiovascular estão associados a diminuição de eventos cardiovasculares e na mortalidade por doença cardiovascular. ${ }^{(75,86)}$ Entretanto, os estudos supracitados são de delineamento transversal o que limita o estabelecimento de causalidade.

No presente estudo, não foi observado associação entre as alterações dos níveis de atividade física total sobre as modificações de pressão arterial braquial, pressão arterial central, rigidez arterial (pressão de pulso, índice de aumento, índice de aumento corrigido por 75bpm e velocidade de onda de pulso) no follow-up. Além disso, também não foi encontrado associação entre as alterações nos níveis de atividade física nas alterações da variabilidade da frequência cardíaca (SDNN, RMSSD, pNN50, alta frequência, baixa frequência e baixa frequência/alta frequência) no follow-up.

Algumas hipóteses podem ser levantadas para explicar esses resultados. Primeiro, os pacientes do presente estudo já apresentavam bom controle pressórico no início do estudo, que pode ter minimizado o potencial efeito do aumento dos níveis de atividade física. Segundo, estudos tem mostrado que programas de exercícios estruturados, de intensidade moderado à vigoroso, são capazes de reduzir a pressão arterial, rigidez arterial e variabilidade da frequência cardíaca em jovens saudáveis, com doença cardiovascular e em pacientes com DAOP. ${ }^{(50,87,88)}$ Como os pacientes do presente estudo diminuíram o tempo despendido em atividade física total e apenas 3,9\% atingiram as recomendações de atividade física, ${ }^{(65)}$ é possível hipotetizar que a atividade física esporádica não seja suficiente para promover alterações nesses indicadores. ${ }^{(81)}$ Assim, pode ser necessário que pacientes com DAOP precisem participar de programas regulares de treinamento com frequência e em uma intensidade adequada para melhorarem os indicadores de saúde cardiovascular, além de aumentar seus níveis de atividade física diários.

Outra possível explicação para os dados encontrados no presente estudo seria que os pacientes já apresentavam disfunção autonômica cardíaca e aumento de rigidez arterial e o tempo de seguimento do estudo ( 2 anos) pode ter sido insuficiente para gerar alterações significativas nesses indicadores. De fato, um estudo longitudinal demonstrou uma associação entre maior tempo gasto em atividade física 
moderado à vigoroso e melhorias na variabilidade da frequência cardíaca na população com síndrome metabólica com seguimento maior do que 2 anos de estudo, indicando que o tempo superior a 2 anos poderia promover respostas positivas nos indicadores de saúde cardiovascular. ${ }^{(89)}$

\subsection{Limitações}

Este estudo apresenta algumas limitações que devem ser mencionadas. Não foram descritos o uso de medicamentos utilizados em termos de tipo e dosagem pelos pacientes, o que dificulta o entendimento entre a interação medicamentosa sobre as variáveis clínicas e de saúde cardiovascular. Tivemos uma perda significativa dos dados de variabilidade da frequência cardíaca devido à presença de arritmias cardíacas ou marcapasso, que pode ter afetado o poder amostral para inferir causa e efeito para essas variáveis. Em alguns pacientes, não foi possível coletar dados de rigidez arterial (velocidade de onda de pulso) por não apresentarem pulso femoral detectável (pulso fraco ou inexistente), para realizar a coleta pela técnica de tonometria de aplanação. O nível de atividade física dos pacientes foram analisadas em apenas duas vezes no seguimento de 2 anos. Muitos pacientes que participaram da visita 1 não retornaram para a visita 2 e isso pode ter incorrido num viés de seleção, visto que os pacientes que retornaram, possivelmente, apresentaram maiores cuidados com a saúde e, consequentemente, melhores valores clínicos. Por fim, o tempo de seguimento de 2 anos pode não ter sido suficiente para observamos mudanças nas variáveis clínicas e de saúde cardiovascular. Desta forma, futuros estudos devem ser realizados com maior tempo de seguimento em pacientes com DAOP. 


\section{CONCLUSÕES}

1. Houve redução significativa dos níveis de atividade física total após 2 anos de seguimento em pacientes com doença arterial obstrutiva periférica;

2. Houve piora da rigidez arterial central, índice tornozelo-braquial e o desvio padrão de todos os intervalos RR após 2 anos de seguimento em pacientes com doença arterial obstrutiva periférica;

3. Não houve associação entre as alterações no nível de atividade física e os indicadores de risco cardiovascular após 2 anos de seguimento em pacientes com doença arterial obstrutiva periférica. 


\section{REFERÊNCIAS}

1. Hirsch AT, Haskal ZJ, Hertzer NR, Bakal CW, Creager MA, Halperin JL, et al.; American Association for Vascular Surgery; Society for Vascular Surgery; Society for Cardiovascular Angiography and Interventions; Society for Vascular Medicine and Biology; Society of Interventional Radiology; ACC/AHA Task Force on Practice Guidelines Writing Committee to Develop Guidelines for the Management of Patients With Peripheral Arterial Disease; American Association of Cardiovascular and Pulmonary Rehabilitation; National Heart, Lung, and Blood Institute; Society for Vascular Nursing; TransAtlantic Inter-Society Consensus; Vascular Disease Foundation. ACC/AHA 2005 Practice Guidelines for the management of patients with peripheral arterial disease (lower extremity, renal, mesenteric, and abdominal aortic): a collaborative report from the American Association for Vascular Surgery/Society for Vascular Surgery, Society for Cardiovascular Angiography and Interventions, Society for Vascular Medicine and Biology, Society of Interventional Radiology, and the ACC/AHA Task Force on Practice Guidelines (Writing Committee to Develop Guidelines for the Management of Patients With Peripheral Arterial Disease): endorsed by the American Association of Cardiovascular and Pulmonary Rehabilitation; National Heart, Lung, and Blood Institute; Society for Vascular Nursing; Trans Atlantic Inter-Society Consensus; and Vascular Disease Foundation. Circulation. 2006;113(11):e463-654.

2. Makdisse M, Pereira AC, Brasil DP, Borges JL, Machado-Coelho GL, Krieger JE, et al.; Hearts of Brazil Study and Peripheral Arterial Disease Committee of the Brazilian Society of Cardiology/Funcor. Prevalence and risk factors associated with peripheral arterial disease in the Hearts of Brazil Project. Arq Bras Cardiol. 2008;91(6):370-82.

3. Norgren L, Hiatt WR, Dormandy JA, Nehler MR, Harris KA, Fowkes FG, et al.; TASC II Working Group. Inter-society consensus for the management of peripheral arterial disease. Int Angiol. 2007;26(2):81-157.

4. Norgren L, Hiatt WR, Dormandy JA, Nehler MR, Harris KA, Fowkes FG; TASC II Working Group. Inter-Society Consensus for the Management of Peripheral Arterial Disease (TASC II). Eur J Vasc Endovasc Surg. 2007;33 Suppl 1:S1-75.

5. Spronk S, White JV, Bosch JL, Hunink MG. Impact of claudication and its treatment on quality of life. Semin Vasc Surg. 2007;20(1):3-9.

6. Gardner AW, Montgomery PS, Scott KJ, Afaq A, Blevins SM. Patterns of ambulatory activity in subjects with and without intermittent claudication. J Vasc Surg. 2007;46(6):1208-14.

7. Gardner AW, Montgomery PS. The effect of metabolic syndrome components on exercise performance in patients with intermittent claudication. J Vasc Surg. 2008;47(6):1251-8.

8. Criqui MH, Langer RD, Fronek A, Feigelson HS, Klauber MR, McCann TJ, et al. Mortality over a period of 10 years in patients with peripheral arterial disease. $\mathrm{N}$ Engl J Med. 1992;326(6):381-6.

9. Steg PG, Bhatt DL, Wilson PW, D'Agostino R Sr, Ohman EM, Röther J, et al.; REACH Registry Investigators. One-year cardiovascular event rates in outpatients with atherothrombosis. JAMA. 2007;297(11):1197-206.

10. Weitz JI, Byrne J, Clagett GP, Farkouh ME, Porter JM, Sackett DL, et al. Diagnosis and treatment of chronic arterial insufficiency of the lower extremities: a critical review. Circulation. 1996;94(11):3026-49. 
11. Chehuen M, Cucato GG, Carvalho CR, Ritti-Dias RM, Wolosker N, Leicht AS, et al. Walking training at the heart rate of pain threshold improves cardiovascular function and autonomic regulation in intermittent claudication: a randomized controlled trial. J Sci Med Sport. 2017;20(10):886-92.

12. Gando Y, Yamamoto K, Murakami H, Ohmori Y, Kawakami R, Sanada K, et al. Longer time spent in light physical activity is associated with reduced arterial stiffness in older adults. Hypertension. 2010;56(3):540-6.

13. Gommans LN, Scheltinga MR, van Sambeek MR, Maas AH, Bendermacher BL, Teijink JA. Gender differences following supervised exercise therapy in patients with intermittent claudication. J Vasc Surg. 2015;62(3):681-8.

14. Rosamond W, legal K, Furie K, Go A, Greenlund K, Haase N, Hailpern SM, Ho M, Howard V, Kissela B, Kittner S, Lloyd-Jones D, McDermott M, Meigs J, Moy C, Nichol G, O'Donnell C, Roger V, Sorlie P, Steinberger J, Thom T, Wilson M, Hong Y; American Heart Association Statistics Committee and Stroke Statistics Subcommittee. Heart disease and stroke statistics2008 update: a report from the American Heart Association Statistics Committee and Stroke Statistics Subcommittee. Circulation. 2008;117(4e25-146).

15. Milani RV, Lavie CJ. The role of exercise training in peripheral arterial disease. Vasc Med. 2007;12(4):351-8.

16. Aboyans V, Ricco JB, Bartelink ME, Björck M, Brodmann M, Cohnert T, et al.; ESC Scientific Document Group. 2017 ESC guidelines on the diagnosis and treatment of peripheral arterial diseases, in collaboration with the European Society for Vascular Surgery (ESVS): document covering atherosclerotic disease of extracranial carotid and vertebral, mesenteric, renal, upper and lower extremity arteriesEndorsed by: the European Stroke Organization (ESO)The Task Force for the Diagnosis and Treatment of Peripheral Arterial Diseases of the European Society of Cardiology (ESC) and of the European Society for Vascular Surgery (ESVS). Eur Heart J. 2018;39(9):763-816.

17. Fowkes FG, Rudan D, Rudan I, Aboyans V, Denenberg JO, McDermott MM, et al. Comparison of global estimates of prevalence and risk factors for peripheral artery disease in 2000 and 2010: a systematic review and analysis. Lancet. 2013;382(9901):1329-40.

18. McDermott MM. Sex differences in the ankle brachial index measurement and interpreting findings of sex differences in peripheral artery disease burden. Circ Cardiovasc Qual Outcomes. 2016;9(2 Suppl 1):S5-7.

19. Becker GJ, McClenny TE, Kovacs ME, Raabe RD, Katzen BT. The importance of increasing public and physician awareness of peripheral arterial disease. J Vasc Interv Radiol. 2002;13(1):7-11.

20. Manfredini F, Malagoni AM, Mascoli F, Mandini S, Taddia MC, Basaglia N, et al. Training rather than walking: the test in -train out program for home-based rehabilitation in peripheral arteriopathy. Circ J. 2008;72(6):946-52.

21. Gardner AW, Montgomery PS. Comparison of three blood pressure methods used for determining ankle/brachial index in patients with intermittent claudication. Angiology. 1998;49(9):723-8.

22. Munger MA, Hawkins DW. Atherothrombosis: epidemiology, pathophysiology, and prevention. J Am Pharm Assoc (2003). 2004;44(2 Suppl 1):S5-12; quis S12-3. 
23. McDermott MM. Ankle brachial index as a predictor of outcomes in peripheral arterial disease. J Lab Clin Med. 1999;133(1):33-40.

24. Criqui MH, Aboyans V. Epidemiology of peripheral artery disease. Circ Res. 2015;116(9):1509-26. Figure 3, Odds ratios for peripheral artery disease in high-income countries (HIC) and low- to middle income countries (LMIC); p. 1518.

25. Fowkes FG, Aboyans V, Fowkes FJ, McDermott MM, Sampson UK, Criqui MH. Peripheral artery disease: epidemiology and global perspectives. Nat Rev Cardiol. 2017;14(3):156-70.

26. Joosten MM, Pai JK, Bertoia ML, Rimm EB, Spiegelman D, Mittleman MA, et al. Associations between conventional cardiovascular risk factors and risk of peripheral artery disease in men. JAMA. 2012;308(16):1660-7.

27. Eraso LH, Fukaya E, Mohler ER 3rd, Xie D, Sha D, Berger JS. Peripheral arterial disease, prevalence and cumulative risk factor profile analysis. Eur J Prev Cardiol. 2014;21(6):704-11.

28. Dua A, Lee CJ. Epidemiology of peripheral arterial disease and critical limb ischemia. Tech Vasc Interv Radiol. 2016;19(2):91-5.

29. Conte SM, Vale PR. Peripheral arterial disease. Heart Lung Circ. 2018;27(4):427-32.

30. Gerage AM, Correia MA, Oliveira PM, Palmeira AC, Domingues WJ, Zeratti AE, et al. Physical activity levels in peripheral artery disease patients. Arq Bras Cardiol. 2019;S0066$782 \times 2019005001306$.

31. McDermott MM, Ferrucci L, Guralnik J, Tian L, Liu K, Hoff F, et al. Pathophysiological changes in calf muscle predict mobility loss at 2-year follow-up in men and women with peripheral arterial disease. Circulation. 2009;120(12):1048-55.

32. McDermott MM, Criqui MH, Greenland P, Guralnik JM, Liu K, Pearce WH, et al. Leg strength in peripheral arterial disease: associations with disease severity and lower-extremity performance. J Vasc Surg. 2004;39(3):523-30.

33. Kropielnicka K, Dziubek W, Bulińska K, Stefańska M, Wojcieszczyk-Latos J, Jasiński R, et al. Influence of the physical training on muscle function and walking distance in symptomatic peripheral arterial disease in elderly. BioMed Res Int. 2018;2018:1937527.

34. Hart CR, Layec G, Trinity JD, Kwon OS, Zhao J, Reese VR, et al. Increased skeletal muscle mitochondrial free radical production in peripheral arterial disease despite preserved mitochondrial respiratory capacity. Exp Physiol. 2018;103(6):838-50.

35. Ernst E, Fialka V. A review of the clinical effectiveness of exercise therapy for intermittent claudication. Arch Intern Med. 1993;153(20):2357-60.

36. Jornal Vascular Brasileiro. Rio de Janeiro: Sociedade Brasileira de Angiologia e Cirurgia Vascular. Vol. 4, No. 3, Suppl 4, 2005.

37. Dormandy JA, Rutherford RB. Management of peripheral arterial disease (PAD). TASC Working Group. TransAtlantic Inter-Society Consensus (TASC). J Vasc Surg. 2000;31(1 Pt 2):S1-296.

38. Nguyen LL, Hevelone N, Rogers SO, Bandyk DF, Clowes AW, Moneta GL, et al. Disparity in outcomes of surgical revascularization for limb salvage: race and gender are synergistic determinants of vein graft failure and limb loss. Circulation. 2009;119(1):123-30. 
39. Millar PJ, Levy AS, McGowan CL, McCartney N, MacDonald MJ. Isometric handgrip training lowers blood pressure and increases heart rate complexity in medicated hypertensive patients. Scand J Med Sci Sports. 2013;23(5):620-6.

40. Munger MA,HawkinsW. Atherothrombosis: epidemiology, pathophysiology, and prevention. J Am Pharm Assoc (2003). 2004;44(2 Suppl 1):S5-12; quis S12-3. Review.

41. Regensteiner JG, Meyer TJ, Krupski WC, Cranford LS, Hiatt WR, Regensteiner JG. Hospital vs home-based exercise rehabilitation for patients with peripheral arterial occlusive disease. Angiology. 1997;48(4):291-300.

42. Gardner AW, Katzel LI, Sorkin JD, Goldberg AP. Effects of long-term exercise rehabilitation on claudication distances in patients with peripheral arterial disease: a randomized controlled trial. J Cardiopulm Rehabil. 2002;22(3):192-8.

43. Cheetham DR, Burgess L, Ellis M, Williams A, Greenhalgh RM, Davies AH. Does supervised exercise offer adjuvant benefit over exercise advice alone for the treatment of intermittent claudication? A randomised trial. Eur J Vasc Endovasc Surg. 2004;27(1):17-23.

44. Treesak C, Kasemsup V, Treat-Jacobson D, Nyman JA, Hirsch AT. Cost-effectiveness of exercise training to improve claudication symptoms in patients with peripheral arterial disease. Vasc Med. 2004;9(4):279-85.

45. Lauret GJ, Fakhry F, Fokkenrood HJ, Hunink MG, Teijink JA, Spronk S. Modes of exercise training for intermittent claudication. Cochrane Database Syst Rev. 2014;(7):CD009638.

46. Selvin E, Erlinger TP. Prevalence of and risk factors for peripheral arterial disease in the United States: results from the National Health and Nutrition Examination Survey, 1999-2000. Circulation. 2004;110(6):738-43.

47. Bhatt DL, Steg PG, Ohman EM, Hirsch AT, Ikeda Y, Mas JL, et al.; REACH Registry Investigators. International prevalence, recognition, and treatment of cardiovascular risk factors in outpatients with atherothrombosis. JAMA. 2006;295(2):180-9.

48. Izquierdo-Porrera AM, Gardner AW, Powell CC, Katzel LI. Effects of exercise rehabilitation on cardiovascular risk factors in older patients with peripheral arterial occlusive disease. J Vasc Surg. 2000;31(4):670-7.

49. Grizzo Cucato G, de Moraes Forjaz CL, Kanegusuku H, da Rocha Chehuen M, Riani Costa $\mathrm{LA}$, Wolosker N, et al. Effects of walking and strength training on resting and exercise cardiovascular responses in patients with intermittent claudication. Vasa. 2011;40(5):390-7.

50. Chehuen MR. [Effect of walking training on cardiovascular risk, function and regulation in patients with intermittent claudication] [thesis]. [Internet]. São Paulo: Universidade de São Paulo, Escola de Educação Física e Esporte; 2014 [cited 2019 Jan 10]. Available from: https://doi.org/10.11606/T.39.2014.tde-11052015-095750. Portuguese.

51. McGuigan MR, Bronks R, Newton RU, Sharman MJ, Graham JC, Cody DV, et al. Resistance training in patients with peripheral arterial disease: effects on myosin isoforms, fiber type distribution, and capillary supply to skeletal muscle. J Gerontol A Biol Sci Med Sci. 2001;56(7):B302-10.

52. Treat-Jacobson D, Bronas UG, Leon AS. Efficacy of arm-ergometry versus treadmill exercise training to improve walking distance in patients with claudication. Vasc Med. 2009;14(3):203-13. 
53. Bronas UG, Treat-Jacobson D, Leon AS. Comparison of the effect of upper body-ergometry aerobic training vs treadmill training on central cardiorespiratory improvement and walking distance in patients with claudication. J Vasc Surg. 2011;53(6):1557-64.

54. Gomes APF, Correia MA, Soares AHG, Cucato GG, Lima AHRA, Cavalcante BR, SobralFilho DC, Ritti-Dias RM. Effects of Resistance Training on Cardiovascular Function in Patients With Peripheral Artery Disease: A Randomized Controlled Trial. J Strength Cond Res. 2018;32(4):1072-1080.

55. Ng PW, Hollingsworth SJ, Luery H, Kumana TJ, Chaloner EJ. Intermittent claudication: exercise-increased walking distance is not related to improved cardiopulmonary fitness. Eur $\mathrm{J}$ Vasc Endovasc Surg. 2005 Oct;30(4):391-4.

56. Zagura M, Kals J, Serg M, Kampus P, Zilmer M, Jakobson M, et al. Structural and biochemical characteristics of arterial stiffness in patients with atherosclerosis and in healthy subjects. Hypertens Res. 2012;35(10):1032-7.

57. Correia MA. Hipotensão pós-exercício de força em indivíduos com doença arterial periférica: análise dos mecanismos hemodinâmicos, autonômicos e vasculares. [dissertação]. [João Pessoa]: Universidade Federal da Paraíba; 2014.

58. Correia MA, Soares AH, Cucato GG, Lima AH, Gomes AP, Prazeres TM, et al. Vascular mechanisms of post-exercise blood pressure responses in peripheral artery disease. Int $\mathrm{J}$ Sports Med. 2015;36(13):1046-51.

59. Walker RD, Nawaz S, Wilkinson CH, Saxton JM, Pockley AG, Wood RF. Influence of upperand lower-limb exercise training on cardiovascular function and walking distances in patients with intermittent claudication. J Vasc Surg. 2000;31(4):662-9.

60. Sandercock GR, Hodges LD, Das SK, Brodie DA. The impact of short term supervised and home-based walking programmes on heart rate variability in patients with peripheral arterial disease. J Sports Sci Med. 2007;6(4):471-6.

61. Leicht AS, Crowther RG, Golledge J. Influence of peripheral arterial disease and supervised walking on heart rate variability. J Vasc Surg. 2011;54(5):1352-9.

62. Parmenter BJ, Raymond J, Fiatarone Singh MA. The effect of exercise on haemodynamics in intermittent claudication: a systematic review of randomized controlled trials. Sports Med. 2010;40(5):433-47.

63. Andreozzi GM, Leone A, Laudani R, Deinite G, Martini R. Acute impairment of the endothelial function by maximal treadmill exercise in patients with intermittent claudication, and its improvement after supervised physical training. Int Angiol. 2007;26(1):12-7.

64. Januszek R, Mika P, Konik A, Petriczek T, Nowobilski R, Niżankowski R. Effect of treadmill training on endothelial function and walking abilities in patients with peripheral arterial disease. $J$ Cardiol. 2014;64(2):145-51.

65. World Health Organization. Global recommendations on physical activity for health. Geneva: World Health Organization; 2010.

66. Barbosa JP, Farah BQ, Chehuen M, Cucato GG, Farias Júnior JC, Wolosker N, et al. Barriers to physical activity in patients with intermittent claudication. Int $\mathrm{J}$ Behav Med. 2015;22(1):70-6.

67. Gardner AW, Montgomery PS, Parker DE. Physical activity is a predictor of all-cause mortality in patients with intermittent claudication. J Vasc Surg. 2008;47(1):117-22. 
68. Garg PK, Tian L, Criqui MH, Liu K, Ferrucci L, Guralnik JM, et al. Physical activity during daily life and mortality in patients with peripheral arterial disease. Circulation. 2006;114(3):2428.

69. Sociedade Brasileira de Cardiologia; Sociedade Brasileira de Hipertensão; Sociedade Brasileira de Nefrologia. VI Diretrizes Brasileiras de Hipertensão. Arq Bras Cardiol. 2010;95(1 supl.1):1-51.

70. Harris RA, Nishiyama SK, Wray DW, Richardson RS. Ultrasound assessment of flowmediated dilation. Hypertension. 2010;55(5):1075-85.

71. Malik M, Bigger JT, Camm AJ, Kleiger RE, Malliani A, Moss AJ, et al.; Task Force of the European Society of Cardiology and the North American Society of Pacing and Electrophysiology. Heart rate variability. Standards of measurement, physiological interpretation, and clinical use. Eur Heart J. 1996;17(3):354-81.

72. Barbosa JP, Henriques PM, Barros MV, Wolosker N, Ritti-Dias RM. Physical activity level in individuals with peripheral arterial disease: a systematic review. J. Vasc Bras. 2012;11(1):22-8.

73. Gardner AW, Montgomery PS, Scott KJ, Afaq A, Blevins SM. Patterns of ambulatory activity in subjects with and without intermittent claudication. J Vasc Surg. 2007;46(6):1208-14.

74. Panico MD, Spichler ES, Neves MF, Pinto LW, Spichler D. Prevalence and risk factors of symptomatic and asymptomatic peripheral arterial disease in a tertiary care hospital, Rio de Janeiro, Brazil. J Vasc Bras. 2009;8(2):125-32.

75. Chobanian AV, Bakris GL, Black HR, Cushman WC, Green LA, Izzo JL Jr, et al.; National High Blood Pressure Education Program Coordinating Committee. The Seventh Report of the Joint National Committee on Prevention, Detection, Evaluation, and Treatment of High Blood Pressure: the JNC 7 report. JAMA. 2003;289(19):2560-72.

76. van Popele NM, Grobbee DE, Bots ML, Asmar R, Topouchian J, Reneman RS, et al. Association between arterial stiffness and atherosclerosis: the Rotterdam Study. Stroke. $2001 ; 32(2): 454-60$.

77. Palombo C, Kozakova M. Arterial stiffness, atherosclerosis and cardiovascular risk: pathophysiologic mechanisms and emerging clinical indications. Vascul Pharmacol. 2016;77:17.

78. Germano-Soares AH, Farah BQ, Andrade-Lima A, Domingues WR, Cavalcante BR, de Almeida Correia M, et al. Factors associated to arterial stiffness in patients With symptomatic peripheral artery disease. Ann Vasc Surg. 2019. pii: S0890-5096(19)30491-1.

79. Goernig M, Schroeder R, Roth T, Truebner S, Palutke I, Figulla HR, et al. Peripheral arterial disease alters heart rate variability in cardiovascular patients. Pacing Clin Electrophysiol. 2008;31(7):858-62.

80. Farah BQ, Andrade-Lima A, Germano-Soares AH, Christofaro DG, de Barros MV, do Prado $W L$, et al. Physical activity and heart rate variability in adolescents with abdominal obesity. Pediatr Cardiol. 2018;39(3):466-72.

81. Gerage AM, Benedetti TR, Farah BQ, Santana FS, Ohara D, Andersen LB, et al. Sedentary behavior and light physical activity are associated with brachial and central blood pressure in hypertensive patients. PLoS One. 2015;10(12):e0146078. 
82. Sugawara J, Otsuki T, Tanabe T, Hayashi K, Maeda S, Matsuda M. Physical activity duration, intensity, and arterial stiffening in postmenopausal women. Am J Hypertens. 2006;19(10):1032-6.

83. Germano-Soares AH, Andrade-Lima A, Menêses AL, Correia MA, Parmenter BJ, Tassitano $\mathrm{RM}$, et al. Association of time spent in physical activities and sedentary behaviors with carotidfemoral pulse wave velocity: a systematic review and meta-analysis. Atherosclerosis. 2018;269:211-8.

84. O'Donovan C, Lithander FE, Raftery T, Gormley J, Mahmud A, Hussey J. Inverse relationship between physical activity and arterial stiffness in adults with hypertension. J Phys Act Health. 2014;11(2):272-7.

85. Sandercock GR, Hardy-Shepherd D, Nunan D, Brodie D. The relationships between selfassessed habitual physical activity and non-invasive measures of cardiac autonomic modulation in young healthy volunteers. J Sports Sci. 2008;26(11):1171-7.

86. Andersson C, Lyass A, Larson MG, Spartano NL, Vita JA, Benjamin EJ, et al. Physical activity measured by accelerometry and its associations with cardiac structure and vascular function in young and middle-aged adults. J Am Heart Assoc. 2015;4(3):e001528.

87. Ciolac EG, Bocchi EA, Bortolotto LA, Carvalho VO, Greve JM, Guimarães GV. Effects of high-intensity aerobic interval training vs. moderate exercise on hemodynamic, metabolic and neuro-humoral abnormalities of young normotensive women at high familial risk for hypertension. Hypertens Res. 2010;33(8):836-43.

88. Heffernan KS, Yoon ES, Sharman JE, Davies JE, Shih YT, Chen CH, et al. Resistance exercise training reduces arterial reservoir pressure in older adults with prehypertension and hypertension. Hypertens Res. 2013;36(5):422-7.

89. Soares-Miranda L, Sandercock G, Vale S, Santos R, Abreu S, Moreira C, et al. Metabolic syndrome, physical activity and cardiac autonomic function. Diabetes Metab Res Rev.

2012;28(4):363-9. 


\begin{abstract}
Introduction: Patients with peripheral obstructive arterial disease present reduced functional capacity and high risk of fatal and nonfatal cardiovascular events. Although exercise is a therapeutic approach to improve functional capacity, little is known about its possible positive effects on longitudinal cardiovascular risk indicators. Purpose: To analyze longitudinally the influence of physical activity level on cardiovascular risk indicators in patients with peripheral obstructive arterial disease. Methods: This study started in 2015. In the first phase, we recruited 226 patients. In the second phase, after 2 years, we included 77 patients who accepted the invitation to return and underwent all evaluations performed in the first evaluation. Levels of physical activity (accelerometer) and indicators of cardiovascular risk (blood pressure, cardiac autonomic modulation and arterial stiffness) were evaluated. Descriptive statistics for continuous variables were expressed as mean, standard deviation, and 95\% confidence interval. Categorical variables were presented as percentages and their $95 \%$ Cls. Median comparisons over time were analyzed using analysis of variance for repeated measures with the Bonferroni post-hoc test. The significance level adopted was 5\%. Results: Patients with peripheral obstructive arterial disease presented worse levels central arterial stiffness showed ankle-brachial index and standard deviation of all RR intervals $(p<0.05)$. In addition, the patients had reduced total physical activity levels $(p<0.05)$. On the other hand, There was no change in cardiovascular risk indicators on time spent on total physical activity $(p>0.05)$. Conclusions: Patients with peripheral obstructive arterial disease we observed reduction total physical activity levels. However, there was no influence of the level of total physical activity on changes in cardiovascular risk indicators in patients with peripheral obstructive arterial disease after 2-year follow-up.
\end{abstract}

Keywords: Peripheral obstructive arterial disease; Cardiovascular system; Arterial pressure; Motor activity 